\title{
Longitudinal wave attenuation in polycrystals with elongated grains: 3D numerical and analytical modeling
}

\author{
M. Huang ${ }^{1, \mathrm{a}}$, G. Sha ${ }^{2}$, P. Huthwaite ${ }^{1}$, S. I. Rokhlin'², and M. J. S. Lowe ${ }^{1}$ \\ ${ }^{1}$ Department of Mechanical Engineering, Imperial College London, Exhibition Road, London \\ SW7 2AZ, United Kingdom \\ ${ }^{2}$ Department of Materials Science and Engineering, Edison Joining Technology Center, The \\ Ohio State University, 1248 Arthur E. Adams Drive, Columbus, OH 43221, United States
}

This work develops a second-order approximation (SOA) model and a three-dimensional (3D) finite element (FE) model to calculate scattering-induced attenuation for elastic wave propagation in polycrystals with elongated grains of arbitrary crystal symmetry. The SOA model accounts for some degree of multiple scattering while the 3D FE model includes all scattering possibilities. The SOA model incorporates the accurate geometric two-point correlation function obtained from the FE material systems to enable comparative studies between the two models. Also, the analytical Rayleigh and stochastic asymptotes are presented to provide explicit insights into propagation behaviors. Quantitative agreement is found between the FE and analytical models for all evaluated cases. In particular, the FE simulations support the SOA model prediction that grain shape does not exert influence on attenuation in the Rayleigh regime and its effect emerges as frequency increases to the stochastic regime, showing anisotropy in attenuation. This attenuation anisotropy intensifies with the increase of frequency, but it exhibits a complicated behavior as frequency transits into the geometric regime. Wavefield fluctuations captured from the FE simulations are provided to help observe these complex scattering behaviors. The proportionality of attenuation to elastic scattering factors is also quantitatively evaluated.

Keywords: elastic wave; polycrystal; grain elongation; second-order approximation; finite element; attenuation

a Electronic mail: m.huang16@imperial.ac.uk

Manuscript accepted for publication in J. Acoust. Soc. Am. 149 (4), April 2021. 


\section{INTRODUCTION}

Scattering arises as an elastic wave propagates through a polycrystalline medium due to elastic tensor fluctuations on the propagation path. Consequently, the coherent part of the propagating wave experiences scattering-induced attenuation and velocity dispersion that depend on the frequency and the geometric and elastic properties of the grains. Polycrystals are random[1] in their nature due to randomness in the crystallographic orientations and the shapes and sizes of grains. Understanding the wave propagation behavior in polycrystals remains a longstanding interest for many practical applications, such as non-destructive evaluation[1-5] and characterization of microstructures[1,2,6-10].

The attenuation and velocity dispersion of waves in polycrystals with statistically equiaxed grains have received extensive attention over the past few decades. Early studies, as reviewed by Papadakis[8], obtained analytical Rayleigh and stochastic asymptotes for statistically equiaxed grains of cubic, hexagonal and orthorhombic symmetries and described experimental results mainly for cubic polycrystals. Significant progress was achieved by Stanke and Kino[11] who, based on the Keller approximation[12,13], developed a unified second-order approximation (SOA) model that is valid for all frequency ranges for statistically equiaxed cubic polycrystals (they have also provided an in-depth overview of the prior theoretical work). An equivalent general model was later formulated by Weaver[14] who employed the first-order smoothing approximation of the Dyson equation[15]. The dispersion equation obtained by Weaver[14] is numerically identical[16] to that of Stanke and Kino[11]; however, the further evaluation and final results by Weaver[14] were obtained by invoking the Born approximation. These models considered untextured single-phase polycrystals with statistically equiaxed grains of cubic crystal symmetry, but they were subsequently extended to a variety of materials with macrotexture, lower crystal symmetries, and duplex microstructures[9,16,25,17-24]. The theoretical models are necessarily approximate, and the Stanke and Kino[11] and Weaver[14] models are accurate to the second order of material inhomogeneity perturbations, allowing for the consideration of partial forward multiple scattering. However, only a few extensions[16,19,22-24] maintained the second-order accuracy in the final results, while others (including Weaver[14]) additionally introduced the Born approximation to deliver explicit expressions for attenuation $[9,17,18,20,21,25]$, limiting their validities to even lower-order scattering in frequencies below 
the geometric regime. Alternatively, important steps of progress were made recently in the finite element (FE) elastodynamic modeling of three-dimensional (3D) polycrystals with statistically equiaxed grains[23,24,26-28]. This method, in contrast to the theoretical models, accounts for all possible multiple scattering events and is not limited by the level of grain scattering in its simulation of wave propagation in polycrystals of realistic sample volumes. Thus, it has enabled the evaluation of the approximations of the theoretical models that show generally good quantitative agreement with the FE models across different frequency ranges, crystal symmetries, inhomogeneity levels, and grain size distributions for polycrystals with statistically equiaxed grains[23,24,26-28].

However, relatively limited studies have been conducted to understand the propagation of waves in polycrystals with statistically elongated grains, despite the fact that the grains of most polycrystals in practice tend to be elongated due to material processing like forging, rolling, and extrusion[2,9,29,30].

Theoretical treatments exist for waves in polycrystals with statistically elongated grains, but a theory maintaining the exact second-order accuracy has been absent so far for grains of arbitrary crystal symmetry. The theoretical analysis was performed for polycrystals with statistically elongated grains of cubic crystal symmetry by Ahmed and Thompson[29] by directly extending and numerically evaluating the Stanke and Kino[11] dispersion equation in its initial general form. This was followed by extensions of the Weaver model to polycrystals of lower crystal symmetry with statistically elongated grains[17,31,32] and elongated microtexture regions[20,21] by Rokhlin and co-authors, but they have invoked the Born approximation and thus limited their analyses to frequencies below the geometric regime. Recently, a far-field approximation (FFA) model was developed by Rokhlin et al.[16] for ellipsoidal grains of general crystallographic symmetry that gives good agreement with the SOA model in all frequency ranges. Also, a spectral function approach for the SOA model was introduced by Calvet and Margerin[33,34] where the attenuation and velocity are obtained by evaluating the spectral function for cubic polycrystals. It is advantageous to explore the second-order model for polycrystals with statistically ellipsoidal grains of arbitrary crystal symmetry and systematically compare it with the FE simulations, and this is the aim of this paper.

Prior studies have found significant differences in scattering behavior between statistically 
equiaxed and elongated polycrystals; most importantly, both the attenuation and phase velocity have shown anisotropic behavior in the transition and stochastic regimes due to the grain elongation[16,17,31-35]. This anisotropic behavior for attenuation was supported by experiments on various titanium alloys[1,10,20]. However, the experimental challenges limit the ability in quantitative comparison with, and validation of, the results of theoretical models, due to the difficulty of determining the microstructure statistics especially for polycrystals with statistically elongated microstructures, and the difficulty of attenuation measurements in covering different frequency scattering regimes[1,8,10,36]. A significant scatter of experimental attenuation data was observed [37] in comparison of results for the Rayleigh, transition, and stochastic frequency ranges from different authors and different samples. The 3D FE method, however, does not suffer from these limitations, because of its full control and knowledge of microstructure statistics and its ability to perform simulations in a large frequency range under plane wave conditions[23]. Thus, an opportunity exists to employ the FE method for a better understanding of wave scattering in polycrystals with statistically elongated grains and for quantitative comparison with the theoretical findings.

To fill in the currently existing void, our prior work[38] on phase velocity dispersion and this work on scattering-induced attenuation seize this opportunity and implement a 3D FE model for the accurate simulation of wave propagation in polycrystals with statistically elongated grains evaluating frequency-dependent anisotropic behavior of attenuation. We are not aware of any other studies that have implemented the 3D FE methods for simulating elastic wave scattering in polycrystals with statistically elongated grains. This work advances a theoretical SOA model and a numerical 3D FE model to interpret the behaviors of waves in polycrystals with statistically elongated grains of arbitrary crystallographic symmetries. It is fundamentally important to directly compare the two models for the evaluation of the applicability of the SOA model and the assessment of the physical relations implied in the SOA model. The comparison relies on the accurate microstructure statistics, characterized by the two-point correlation (TPC) function, obtained from the simulated FE material systems in different propagation directions to insert into the SOA model. Contrasting to the TPC function for polycrystals with statistically equiaxed grains[23,24,28], that is of the scalar nature, the accurate incorporation of the spatial, direction-dependent TPC statistics for polycrystals with statistically elongated grains is challenging. The difficulties are accurately measuring the TPC data from FE material models and 
correctly representing the measured data by a generalized TPC function that is used in the theoretical models. This work reports on key approaches addressing these difficulties.

Based on the comparative studies of the theoretical and FE models, this work examines the relationship between the grain elongation and the frequency-dependent directional behavior of scattering-induced attenuation. We demonstrate that the frequency and directional behavior of the attenuation obtained from both the SOA and the FE models show proportionalities to, and thus are scaled by, the two elastic scattering factors in the Rayleigh and stochastic regimes; these findings are consistent with our prior studies[16,24]. They are practically significant since they indicate that the elastic properties of the materials mainly affect wave propagation behaviors only through the two combinations of elastic constants even for triclinic polycrystals with 21 independent elastic constants. The effect of grain elongation on the behavior of phase velocity dispersion has been recently reported in the related comparative study[38].

The polycrystals addressed in this work are composed of single-phase, densely-packed, fully-bonded grains with a statistically elongated shape. In practice, grain elongation is usually accompanied by preferred crystallographic orientation, but considering both aspects adds complexity to the analysis of the grain elongation effect [17]. To isolate the effect of grain shape, we exclude the preferred crystallographic orientation (macrotexture) and assume the uniform random distribution of crystallographic orientations of grains. Therefore, in this study statistical anisotropy of the addressed polycrystals is attributed solely to the grain elongation: the statistical anisotropy is represented in the analytical model by an angle-dependent TPC function. Whereas this simplification is not suitable in all applications, it is an essential first step in order to see the effects of grain geometry without the influence of crystallographic macrotexture. In the presented comparative study, we have selected a statistically ellipsoid-of-rotation shape of grains with an elongation ratio of five, meaning that the average grain radius in the elongated direction is five times that of the shortened direction. However, the theoretical and numerical models established in this work are suitable for general cases and we believe the conclusions and the wave mechanisms revealed in this work are universally applicable to grains with different aspect ratios.

The SOA model, the Born approximation, and the Rayleigh and stochastic asymptotes are described in Sec. II for polycrystals with statistically elongated grains of arbitrary crystal 
symmetry. Sec. III implements the 3D FE model for wave propagation in polycrystals with statistically elongated grains. A quantitative comparison of the SOA and FE models is provided in Sec. IV, in which the influences of grain elongation and elastic properties are discussed for attenuation. The conclusions are given in Sec. V.

\section{SOA MODEL FOR ELASTIC WAVE PROPAGATION IN POLYCRYSTALS WITH ELONGATED GRAINS}

The SOA model for elastic wave propagation in polycrystals with statistically elongated grains has been described in our prior work[38]. Therefore, in addition to some new discussions, only an overview of the essential steps and final equations of the model is provided below.

The propagation of an elastic wave in a statistically anisotropic polycrystal as considered in this work can be approximately solved by replacing the polycrystal with a continuous random medium with small spatial fluctuations of elastic tensor and considering an ensemble-averaged mean field[11,14]. The continuous random medium is related to the original polycrystal by the spatial TPC function. The propagating wave scatters on the elastic perturbations resulting in the perturbed effective wave vector $\mathbf{k}$ of the mean field, which satisfies the dispersion equation[14,23,38]

$$
\omega^{2}-k^{2} V_{0 M}^{2}-m_{M}(\mathbf{k} ; \omega)=0
$$

where $\omega=2 \pi f$ is the angular frequency and $f$ is the frequency. The unit vector $\mathbf{p}$ of the perturbed wave vector, $\mathbf{k}=k \mathbf{p}$, represents the wave propagation direction as illustrated in Fig. 1 . $m_{M}(\mathbf{k} ; \omega)$ is the mass operator, which will be considered and defined below. Note that the incident wave mode $M$ is implicit in the wave number $k$ and $M$ can be any of the three independent modes: longitudinal (L), fast transverse (T1), and slow transverse (T2). $V_{0 M}$ represents the phase velocity of the wave $M$ in the reference medium [23], whose elastic tensor is the Voigt average of the polycrystal. The reference medium is isotropic for the polycrystals considered in this work, and therefore $V_{0 M}$ is independent of the wave propagation direction p.

The mass operator $m_{M}(\mathbf{k} ; \omega)$ in Eq. (1) accounts for the scattering events occurring in the 
polycrystal. This operator cannot be obtained exactly even for scalar cases[12,39], and approximations are generally needed for the formulation. The SOA model in this work adopts the first-order smoothing approximation [14,15] (or equivalently the Bourret approximation $[15,39,40])$ which accounts for double scattering inside each heterogeneity without recurrent visits[23,39] and the corresponding forward scattering events. The model is applicable to all frequency ranges, but it is generally assumed[11,14] that its validity is limited to the cases of small inhomogeneity perturbations of the homogeneous reference medium. The inhomogeneity perturbations result in the perturbations of the incident coherent wavefield and the wave number due to scattering, which is related in this work to the elastic scattering factors $Q$ (the equivalent factor is denoted $\varepsilon^{2}$ in Refs. [11-13] and it is deemed small when $\left.\varepsilon^{2}<<1[11,32]\right)$. Also, it is assumed [11,14] that the propagation distance is relatively small, so the ratio between the incoherent and coherent fields is much smaller than unity [41]. However, recent 3D FE studies $[23,24,27,28]$ have shown that the SOA model is in very good agreement with $3 \mathrm{D}$ FE simulations for polycrystals with statistically equiaxed grains of high grain anisotropy and inhomogeneity perturbations, such as Inconel, copper and triclinic CSP (in this paper we extend those conclusions to polycrystals with statistically elongated grains). The wave propagation distances in those 3D FE simulations are on the order of 10 in terms of average grain diameter and wavelength (this translates to about $10 \mathrm{~mm}$ in studied metals at the frequency of about 6 $\mathrm{MHz})$.

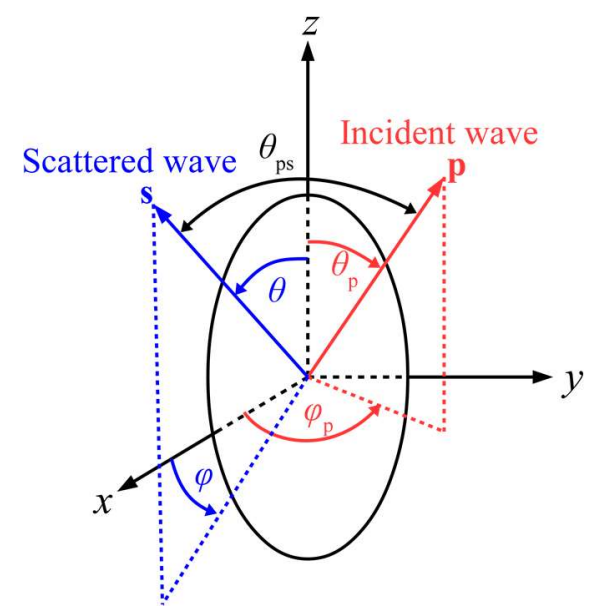

Fig. 1. (Color online) Coordinate system for wave propagation and scattering in polycrystals with statistically ellipsoidal grains. $(x, y, z)$ is the global coordinate system and its axes are aligned with the radii of the ellipsoidal grains. Incident wave propagates in the direction of 
$\mathbf{p}=\left(\sin \theta_{\mathrm{p}} \cos \varphi_{\mathrm{p}}, \sin \theta_{\mathrm{p}} \sin \varphi_{\mathrm{p}}, \cos \theta_{\mathrm{p}}\right)$ and scattered wave in the direction of $\mathbf{s}=(\sin \theta \cos \varphi, \sin \theta \sin \varphi, \cos \theta)$. The angle between the incident and scattered waves is $\theta_{\mathrm{ps}}$.

The mass operator is expressed as $m_{M}(\mathbf{k} ; \omega)=\sum_{N=\{\mathrm{L}, T\}} m_{M \rightarrow N}(\mathbf{k} ; \omega)$ with each component $m_{M \rightarrow N}$ representing the scattering of the wave $M$ to the wave $N$. Note that the $m_{M \rightarrow \mathrm{T}}$ component has collectively considered the scattered $\mathrm{T} 1$ and $\mathrm{T} 2$ waves, which have approximately the same velocities but mutually orthogonal polarizations due to the isotropy of the reference medium[33]. By using the first-order smoothing approximation, the component $m_{M \rightarrow N}$ in the spherical coordinate system is given by[38]

$$
\begin{gathered}
m_{M \rightarrow N}(\mathbf{k} ; \omega)=\frac{k^{2} k_{0 N}^{3}}{\eta \rho^{2} V_{0 N}^{2}}\left\{\mathrm{P} . \mathrm{V} \cdot \int_{0}^{\infty}\left[\frac{\xi^{4}}{1-\xi^{2}} \int_{0}^{\pi} \int_{0}^{2 \pi} f_{M \rightarrow N}(k, \omega, \xi, \theta, \varphi) \sin \theta \mathrm{d} \varphi \mathrm{d} \theta\right] \mathrm{d} \xi\right. \\
\left.-i \frac{\pi}{2} \int_{0}^{\pi} \int_{0}^{2 \pi} f_{M \rightarrow N}(k, \omega, \xi=1, \theta, \varphi) \sin \theta \mathrm{d} \varphi \mathrm{d} \theta\right\}
\end{gathered}
$$

in which the mass density $\rho$ is constant for the polycrystals composed of single-phase grains. $k_{0 N}$ is the wave number for the scattered mode $N$ in the reference medium. P.V. represents Cauchy principal value and $\xi$ is a dimensionless variable. The coefficient $\eta$ is 1 and 2 for longitudinal ( $M=\mathrm{L})$ and transverse ( $M=\mathrm{T}$ ) propagating waves, respectively.

The factor $f_{M \rightarrow N}$ in Eq. (2) can be factorized as[38]

$$
f_{M \rightarrow N}(k, \omega, \xi, \theta, \varphi)=\operatorname{IP}_{M \rightarrow N}(\theta, \varphi) W_{M \rightarrow N}(k, \omega, \xi, \theta, \varphi)
$$

where the inner product $\operatorname{IP}_{M \rightarrow N}$ accounts for the elastic statistics of the polycrystal as well as the propagation and polarization directions of both incident and scattered waves, while the spectral TPC function $W_{M \rightarrow N}$ considers the spatial properties of the polycrystal. The validity of this factorization has been numerically demonstrated for polycrystals with statistically equiaxed grains (statistically isotropic case) [42] and indirectly supported by our prior phase velocity results for polycrystals with ellipsoidal grains (statistically anisotropic case) [38]; further evidence can be found from the attenuation results in Sec. IV of the present work. The inner product is given by $[16,17,38]$

$$
\mathrm{IP}_{M \rightarrow N}(\theta, \varphi)=A_{M N}+B_{M N} \cos ^{2} \theta_{\mathrm{ps}}+C_{M N} \cos ^{4} \theta_{\mathrm{ps}}
$$


where the invariants $A_{M N}, B_{M N}$ and $C_{M N}(M, N \in\{\mathrm{L}, \mathrm{T}\})$ are the sums of the quadratic terms of the elastic constants [17] and their expressions are given in Appendix A for arbitrary single-crystal symmetries. $\theta_{\mathrm{ps}}$ is the angle between the incident and scattered waves and $\cos \theta_{\mathrm{ps}}=\mathbf{p} \cdot \mathbf{s}=\cos \left(\varphi-\varphi_{\mathrm{p}}\right) \sin \theta \sin \theta_{\mathrm{p}}+\cos \theta \cos \theta_{\mathrm{p}}$. The spectral TPC function $W_{M \rightarrow N}$ is given by[38]

$$
W_{M \rightarrow N}(k, \omega, \xi, \theta, \varphi)=\sum_{i=1}^{n} \frac{A_{i} a_{x}^{i} a_{y}^{i} a_{z}^{i}}{\pi^{2}\left[1+q_{x}^{2}\left(a_{x}^{i}\right)^{2}+q_{y}^{2}\left(a_{y}^{i}\right)^{2}+q_{z}^{2}\left(a_{z}^{i}\right)^{2}\right]^{2}},
$$

where $\left(q_{x}, q_{y}, q_{z}\right)=k\left(\sin \theta_{\mathrm{p}} \cos \varphi_{\mathrm{p}}, \sin \theta_{\mathrm{p}} \sin \varphi_{\mathrm{p}}, \cos \theta_{\mathrm{p}}\right)-\xi k_{0 N}(\sin \theta \cos \varphi, \sin \theta \sin \varphi, \cos \theta)$ is the difference between the incident and scattered wave vectors. Eq. (5) is obtained by Fourier transforming the spatial TPC function $w\left(\mathbf{r}=\mathbf{x}-\mathbf{x}^{\prime}\right)$ that describes the probability of two points $\mathbf{x}$ and $\mathbf{x}^{\prime}$ separated by a vector $\mathbf{r}=\mathbf{x}-\mathbf{x}^{\prime}$ being in the same grain. Since the average shape of the elongated grains is considered to be ellipsoidal and the ellipsoidal axes are aligned with the axes of the global coordinate system as shown in Fig. 1, we have assumed the following form for the spatial TPC function[38]

$$
w(\mathbf{r})=\sum_{i=1}^{n} A_{i} \exp \left(-\sqrt{\frac{r_{x}^{2}}{\left(a_{x}^{i}\right)^{2}}+\frac{r_{y}^{2}}{\left(a_{y}^{i}\right)^{2}}+\frac{r_{z}^{2}}{\left(a_{z}^{i}\right)^{2}}}\right),
$$

where $a_{x}^{i}, a_{y}^{i}, a_{z}^{i}$, and $A_{i}$ are the coefficients of the $i$-th term of the exponential series, which are determined by fitting the actual TPC data of the polycrystal. Eq. (6) is a generalization of the single term TPC used for statistically elongated polycrystals in the past $[6,10,16,31,43]$. The applicability of the assumed TPC function, Eq. (6), will be demonstrated below for the FE material models in Sec. III and by the data on the anisotropy of the phase velocity[38]. Note that we do not assign physical meaning to the individual terms of the series and only the total TPC function $w(\mathbf{r})$ describes the polycrystal statistics[23]. However, some insights can be gained from the spectral representation in Eq (5) that each term essentially modifies the spatial spectrum of the actual TPC, eventually representing it with the sum of the individual terms.

Solving Eq. (1) leads to the solution to the perturbed wave number $k$, and the attenuation coefficient and phase velocity can thus be obtained as $\alpha_{M}=\operatorname{Im} k$ and $V_{M}=\omega / \operatorname{Re} k$. Those parameters depend on the wave propagation direction $\mathbf{p}$ due to the form of the TPC function in 
Eq. (6) and its transform in Eq. (5) (physically it is due to the shape of the grains) and are thus statistically anisotropic, even though the reference medium is considered isotropic. The propagation in a random medium with both statistical grain elongation and preferred crystallographic orientation (macroscopic texture) is treated in Ref. [17].

Born approximation. The Born approximation of the SOA model is useful since it provides an explicit expression for attenuation and allows a simple derivation of the Rayleigh and stochastic attenuation asymptotes given below. In the Born approximation, the perturbed wave vector $\mathbf{k}$ in the mass operator, Eq. (2), is replaced by the unperturbed reference wave vector $\mathbf{p} k_{0 M}$. And the small wave number perturbation $\left(\omega / V_{0 M}\right)^{2}-k^{2}$ in the dispersion equation, Eq. (1), is substituted by the first-order term $2 k_{0 M}\left(k_{0 M}-k\right)$ of its Taylor series about $k=k_{0 M}$. These simplifications result in the explicit solution of the perturbed wave number

$$
k=k_{0 M}-\frac{m_{M}\left(\mathbf{p} k_{0 M} ; \omega\right)}{2 k_{0 M} V_{0 M}^{2}}
$$

where the mass operator is given by $m_{M}\left(\mathbf{p} k_{0 M} ; \omega\right)=\sum_{N=\mathrm{L}, \mathrm{T}} m_{M \rightarrow N}\left(\mathbf{p} k_{0 M} ; \omega\right)$. The component $m_{M \rightarrow N}\left(\mathbf{p} k_{0 M} ; \omega\right)$ is obtained by replacing the wave vector $\mathbf{k}$ of Eq. (2) by $\mathbf{p} k_{0 M}$. Due to this replacement, the real and imaginary parts of the wave number can be separated. The real part determines the phase velocity as given in our prior work[38] and the imaginary part delivers the attenuation coefficient

$$
\alpha_{M}=\sum_{N=\mathrm{L}, \mathrm{T}} \alpha_{M \rightarrow N}
$$

where

$$
\alpha_{M \rightarrow N}=\frac{\pi \omega^{4}}{4 \eta \rho^{2} V_{0 M}^{3} V_{0 N}^{5}} \int_{0}^{\pi} \int_{0}^{2 \pi} f_{M \rightarrow N}\left(k_{0 M}, \omega, \xi=1, \theta, \varphi\right) \sin \theta \mathrm{d} \varphi \mathrm{d} \theta .
$$

The Born approximation only considers single scattering. This limits its validity to frequencies below the geometric regime[11].

To evaluate its range of validity, it is compared with the SOA model in Fig. 2 for longitudinal wave attenuation in polycrystalline Copper Sulfate Pentahydrate (CSP, $\rho=2286 \mathrm{~kg} / \mathrm{m}^{3}$, $V_{0 \mathrm{~L}}=4874.33 \mathrm{~m} / \mathrm{s}, \quad V_{0 \mathrm{~T}}=2303.21 \mathrm{~m} / \mathrm{s}$, elastic constants in Ref. [24]) with statistically 
elongated grains of triclinic crystal symmetry. The geometric TPC function $w(\mathbf{r})$ of the material is determined from the FE material models as presented in Sec. III and the expression of the function is given by Eq. (6) with the coefficients being listed in Table III of our prior work [38]. In this example, the elongated grains have an elongation ratio of 5, and the mean grain radius in the elongated $z$-direction is 5 times that of the shortened $x$-direction, i.e., $a_{z}=5 a_{x}$. Here the mean grain radius is represented by the mean line intercept that is determined from the slope[43] at the origin of the TPC function. In the direction with an angle $\theta_{\mathrm{p}}$ to the elongated $z$-direction, the mean grain radius is given by

$$
a_{\theta_{\mathrm{p}}}=-1 /\left[\partial w(\mathbf{r}=\mathbf{0}) / \partial r_{\theta_{\mathrm{p}}}\right]
$$

where $r_{\theta_{\mathrm{p}}}$ denotes the correlation distance at the angle $\theta_{\mathrm{p}}$. As a result, the mean grain radius in the shortened direction is given by

$$
a_{x}=-1 /\left[\partial w(\mathbf{r}=\mathbf{0}) / \partial r_{x}\right]=1 / \sum_{i=1}^{8}\left(A_{i} / a_{x}^{i}\right)=0.22 \mathrm{~mm},
$$

for the example material. The theoretical results are given for both the shortened and elongated directions in the figure, and for both directions $a_{x}$ is used as the normalization factor. The SOA model and Born approximation are numerically implemented and solved in MATLAB[44]. In particular, the transcendental dispersion equation, Eq. (1), of the SOA model is solved with a variant of Newton's method, and the Cauchy integral in Eq. (2) is numerically evaluated with a Gauss-Legendre quadrature method[45]. The computation time for the SOA curve in Fig. 2 is about ten times that for the Born approximation.

The figure shows that the Born approximation agrees very well with the SOA model in low frequencies, and the difference between the two models increases as frequency approaches the geometric regime. The frequency range of validity of the Born approximation for the attenuation is consistent with that for the phase velocity[38].

Rayleigh attenuation asymptote. In the low-frequency Rayleigh scattering regime, the expression for the attenuation of longitudinal and transverse waves is simplified. At this low-frequency limit, the wavelength is much larger than the average grain size in any direction of a polycrystal. By considering this fact, the asymptote for attenuation is obtained that provides 
a better understanding of the scattering phenomenon at a long wavelength. The asymptote is derived in Appendix B from the Born approximation as discussed above. For polycrystals with elongated grains of statistically ellipsoidal shape, the attenuation asymptote is given by

$$
\alpha_{M}^{\mathrm{R}}=\frac{1}{2 \pi} k_{0 M}^{4} V_{\mathrm{eff}}^{\mathrm{g}}\left(Q_{M M}^{*}+\frac{V_{0 M}^{3}}{V_{0 N}^{3}} Q_{M \rightarrow N}\right),
$$

where $M, N \in\{\mathrm{L}, \mathrm{T}\}$ and $M \neq N . V_{\mathrm{eff}}^{\mathrm{g}}=\int_{V} w(\mathbf{r}) \mathrm{d} v$ is the effective grain volume defined by the volumetric integral of the TPC function[11,14] and it is given by $V_{\mathrm{eff}}^{\mathrm{g}}=8 \pi \sum_{i} A_{i} a_{x}^{i} a_{y}^{i} a_{z}^{i}$ for the TPC function in Eq. (6). $Q_{M \rightarrow N}$ is the mode-converted scattering factor and $Q_{M M}^{*}$ is a factor introduced to simplify the above equation; their expressions are given in Appendix A. An example Rayleigh asymptote, Eq. (12), is provided in Fig. 2.

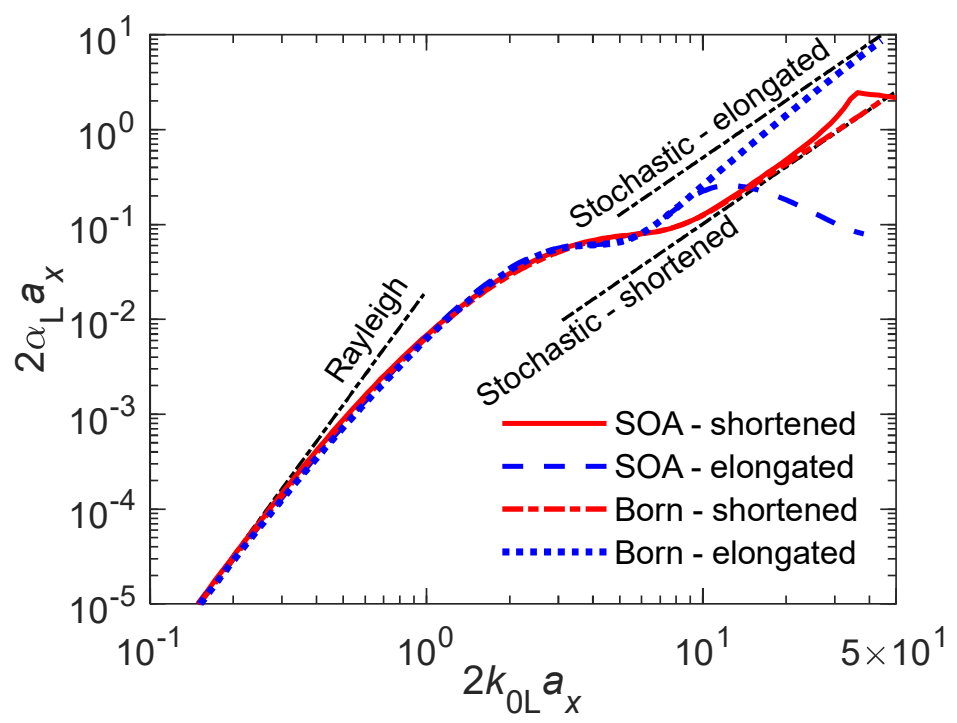

Fig. 2. (Color online) Comparison of the SOA and Born approximation models in all frequency regimes for the normalized attenuation of longitudinal waves. The results are given for polycrystalline CSP (material properties in Ref. [24]) with statistically elongated grains having an elongation ratio of 5. The geometric TPC function of the polycrystal is provided by Eq. (6) with coefficients being given in Table III of our prior work [38]. The mean line intercept grain radius in the shortened $x$-direction is $a_{x}=0.22 \mathrm{~mm}$ and that of the elongated $z$-direction is $a_{z}=5 a_{x}$. The Rayleigh and stochastic asymptotes are obtained from Eqs. (12) and (13), respectively. 
Eq. (12) shows that at the low-frequency limit the attenuation obeys a fourth-power dependence on frequency (frequency is implied in the wave number $k_{0 M}$ ). It is proportional to effective grain volume and thus independent of grain shape, meaning that the attenuation is the same in different wave propagation directions as that for the statistically equiaxed grains[24]. Attenuation also depends on the elastic properties of grains primarily through the scattering factors $Q_{M M}^{*}$, $Q_{M \rightarrow N}$, and wave speeds in the reference medium. For the longitudinal waves considered in this work, it is worth noting that the factor $Q_{M M}^{*}$ in Eq. (12) is small compared to $Q_{M \rightarrow N} V_{0 M}^{3} / V_{0 N}^{3}$. Thus, the longitudinal attenuation is approximately proportional to $Q_{\mathrm{L} \rightarrow \mathrm{T}}$ and the third power of velocity ratio $V_{0 \mathrm{~L}} / V_{0 \mathrm{~T}}$ at the low-frequency limit which is useful for material property normalization as discussed below.

Stochastic attenuation asymptote. The stochastic attenuation asymptote has been derived by Yang et al.[31] and provided in a normalized form by Li and Rokhlin[17] for polycrystals with statistically elongated grains with TPC statistics being represented by a single-term exponential function (i.e., the summation over $i$ in Eq. (6) is not present). The derivation was based on the facts that the same mode scattering is dominant at the stochastic limit, namely $\alpha_{M}^{\mathrm{S}} \approx \alpha_{M \rightarrow M}$, and the forward scattering is dominant in $\alpha_{M \rightarrow M}$. The derivation is not repeated here; readers are referred to Yang et al.[31] for details. Here we follow the normalized form of Li and Rokhlin[17] and adapt it to our case of arbitrary crystallographic symmetry and multi-term generalized TPC function as follows

$$
\alpha_{M}^{\mathrm{S}}=k_{0 M}^{2} a_{\mathrm{eff}}\left(\theta_{\mathrm{p}}, \varphi_{\mathrm{p}}\right) Q_{M \rightarrow M}
$$

where $Q_{M \rightarrow M}$ is the elastic scattering factor and its expression is given in Appendix A. $a_{\text {eff }}\left(\theta_{\mathrm{p}}, \varphi_{\mathrm{p}}\right)$ is the effective correlation length[31] in the direction of wave propagation $\left(\theta_{\mathrm{p}}, \varphi_{\mathrm{p}}\right)$ as illustrated in Fig. 1, and for the generalized TPC function it is given by

$$
a_{\mathrm{eff}}\left(\theta_{\mathrm{p}}, \varphi_{\mathrm{p}}\right)=\sum_{i=1}^{n} \frac{A_{i}}{\sqrt{\frac{\sin ^{2} \theta_{\mathrm{p}} \cos ^{2} \varphi_{\mathrm{p}}}{\left(a_{x}^{i}\right)^{2}}+\frac{\sin ^{2} \theta_{\mathrm{p}} \sin ^{2} \varphi_{\mathrm{p}}}{\left(a_{y}^{i}\right)^{2}}+\frac{\cos ^{2} \theta_{\mathrm{p}}}{\left(a_{z}^{i}\right)^{2}}}} .
$$

Additionally, attenuation also depends on elastic scattering factor $Q_{M \rightarrow M}$ and effective 
correlation length $a_{\mathrm{eff}}\left(\theta_{\mathrm{p}}, \varphi_{\mathrm{p}}\right)$. It is important to note that for statistically ellipsoid-of-rotation grains, attenuation asymptote only changes with angle $\theta_{\mathrm{p}}$ as correlation length $a_{\text {eff }}$ is now a univariate function of $\theta_{\mathrm{p}}$.

Stochastic asymptote is provided in Fig. 2. Eq. (13) demonstrates that in this regime the attenuation obeys a second-power dependence on frequency at the high-frequency stochastic limit. It is clear from Fig. 2 that the stochastic asymptote for the elongated direction approaches the Born approximation only at frequencies significantly above the geometric region transition for the SOA model. This suggests that in this case the stochastic regime is not observed for propagation in the grain elongation direction (it is barely developed in the shortened propagation direction).

\section{FE MODEL FOR ELASTIC WAVES IN POLYCRYSTALS WITH ELONGATED GRAINS}

The FE method for simulating elastic waves in polycrystals with statistically elongated grains was implemented in our prior work[38] so the details are not repeated here. Alternatively, a brief overview of this method is given below, and several previously un-visited aspects are discussed.

The FE method creates a numerical polycrystal model in the Neper program[46] using Voronoi tessellation and then imports the model into our MATLAB[44] toolbox for further processing as described below. The created cuboid model has a volume of $d_{x} \times d_{y} \times d_{z}$ and is packed with non-overlapping and fully bonded grains as represented by convex polyhedra. Example grains are shown in Fig. 3(a). The grain diameters, defined as the cubic root of grain volume [26,27] or the equivalent spherical diameter [28], have a Gaussian distribution. The grains are statistically equiaxed since the Poisson point process is used to randomly place Voronoi seeds in the 3D model volume $[23,24,26,27]$. We have verified that the geometric TPC function of the created model is practically identical in different directions, confirming that the grains are statistically equiaxed.

The polycrystal model is then scaled such that the constituting grains are statistically elongated. This is achieved in our MATLAB toolbox by scaling the coordinates of all polyhedral vertices 
along the three coordinate axes using the following scale factors

$$
S_{x}=S_{y}=1 / \sqrt[3]{R}, \quad S_{z}=R / \sqrt[3]{R}
$$

where $R=S_{z} / S_{x}$ is the elongation ratio. Since the product of the three factors is unity $\left(S_{x} \cdot S_{y} \cdot S_{z}=1\right)$, the volume of each grain remains unchanged during the scaling; this is to achieve a direct comparison of the equiaxed and elongated cases. The resulting model possesses the volume of $d_{x} S_{x} \times d_{y} S_{y} \times d_{z} S_{z}$ and the grains have a statistically ellipsoidal shape as verified below by the measured TPC function. Fig. 3(b) displays typical elongated grains that are obtained by scaling the equiaxed grains in (a) using an elongation ratio of $R=5$. Additionally, the major axis of the elongated model is rotated about the $x$-axis with an angle of $\theta_{\mathrm{p}}$, as illustrated in Fig. 3(b), to simulate the propagation of waves in different directions relative to the elongated direction. We note that the initial polycrystal model is created with a sufficiently large volume such that the rotated model can be cropped to have the dimensions of $d_{x} S_{x} \times d_{y} S_{y} \times d_{z} S_{z}$ along the coordinate axes. The models generated for this work are summarized in Table I.

To simulate a single-phase polycrystal, the grains of each model are assigned with the same material density and elastic properties. The materials used in this work include: the cubic Aluminum $\left(\rho=2700 \mathrm{~kg} / \mathrm{m}^{3}, V_{0 \mathrm{~L}}=6317.52 \mathrm{~m} / \mathrm{s}, V_{0 \mathrm{~T}}=3128.13 \mathrm{~m} / \mathrm{s}\right.$, elastic constants in Ref. [23]) and Inconel ( $\rho=8260 \mathrm{~kg} / \mathrm{m}^{3}, V_{0 \mathrm{~L}}=6025.37 \mathrm{~m} / \mathrm{s}, V_{0 \mathrm{~T}}=3365.54 \mathrm{~m} / \mathrm{s}$, elastic constants in Ref. [23]), and the triclinic copper sulfate pentahydrate (CSP, $\rho=2286 \mathrm{~kg} / \mathrm{m}^{3}$, $V_{0 \mathrm{~L}}=4874.33 \mathrm{~m} / \mathrm{s}, V_{0 \mathrm{~T}}=2303.21 \mathrm{~m} / \mathrm{s}$, elastic constants in Ref. [24]). The elastic scattering factors of the three materials are provided in Table II. In order for a model to be crystallographically isotropic on the macro scale, the crystallographic axes of its grains are uniformly randomly oriented. Our prior work[27] has evaluated and demonstrated that an initially created FE material system with statistically equiaxed grains is absent of crystallographic macrotexture. Since the statistically elongated polycrystals are obtained by geometrically stretching the shape of the grains without changing the crystallographic orientations, they are also free from crystallographic macrotexture. As a result, the anisotropy exhibited in the attenuation (and the phase velocity[38]) stems solely from the statistical grain elongation. 


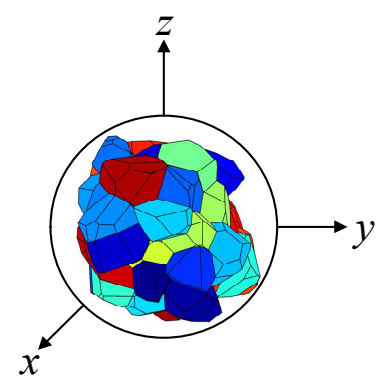

(a) Equiaxed case

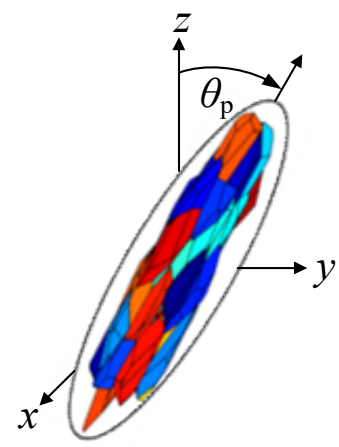

(b) Elongated case

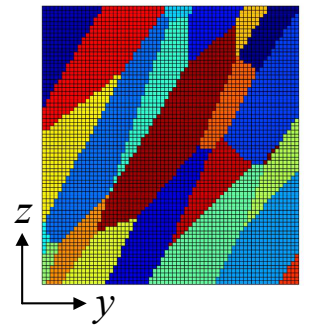
(b) elongated grains and their discretized cross-sections. The elongated grains in (b) are obtained by scaling the equiaxed ones in (a) using the scale factors of $\left(S_{x}, S_{y}, S_{z}\right)=(1,1, R) / \sqrt[3]{R} . R$ is the elongation ratio and in this case $R=5$. Following the scaling, the elongated grains as a whole are rotated about the $x$-axis with an angle of $\theta_{\mathrm{p}}$. The grains in (a) and (b) are subdivided into uniform 8-node linear "brick" elements, and the figure gives cross-sectional views of the elements. These illustrations are chosen to show just a local region; the actual models used in this work have a very much larger number of grains.

The generated models are spatially discretized using structured meshes that subdivide a spatial model into uniform 8-node linear "brick" elements. Example cross-sections of the 3D meshes are illustrated in Fig. 3(a) and (b) for statistically equiaxed and elongated grains, respectively. The element sizes $h$ of the used models are provided in Table I, which are chosen to be smaller than one-tenth of the simulated wavelength in order to minimize numerical errors [26,47]. Additionally, the element size is chosen to be twenty times smaller than the average grain diameter $D$ (the cubic root of average grain volume) to well represent the grains. For statistically equiaxed grains, our previous study[26] suggests that this choice makes the structured mesh perform equally as well as an unstructured mesh that is capable of perfectly describing planar grain boundaries. For statistically elongated grains, we have utilized grain cross-sectional area as a measure to identify the difference of grain geometry before and after discretization. We have found, for all FE models in this work, that this measure is statistically very well preserved after discretization of the created microstructure in comparison to that before discretization. This means that the choice of mesh type and size in this work has delivered a very 
good representation of the statistically elongated grains by structured meshes. Nonetheless, it is possible that there might be a few very sharp tips in the elongated direction that can hardly be represented by structured meshes. However, the possibly-missed sharp tips do not affect scattering results because the mesh size is at least 20 times smaller than the mean grain size in this work.

Table I. Polycrystalline models with elongated grains. Elongation ratio $R$, dimensions $d_{x} \times d_{y} \times d_{z}(\mathrm{~mm})$, the average edge sizes of the grains $D_{x} \times D_{y} \times D_{z}(\mathrm{~mm})$, number of grains $N$, mesh size $h(\mathrm{~mm})$, degree of freedom d.o.f., center frequency for transmitted waves $f_{\mathrm{c}}$ $(\mathrm{MHz})$. The parameters for the respective models with equiaxed grains can be calculated from the given parameters or can be found from Ref. [38].

\begin{tabular}{llllllllll}
\hline \hline & $R$ & $d_{x} \times d_{y} \times d_{z}$ & $D_{x} \times D_{y} \times D_{z}$ & $N$ & $h$ & d.o.f. & \multicolumn{3}{c}{$f_{\mathrm{c}}$} \\
\cline { 6 - 9 } & & & & & & Al & Inconel & CSP \\
\hline N11520 & 5 & $7.02 \times 7.02 \times 29.24$ & $0.29 \times 0.29 \times 1.46$ & 11520 & 0.025 & $278 \times 10^{6}$ & 5,10 & 2,5 & 2,5 \\
N16000 & 5 & $11.70 \times 11.70 \times 14.62$ & $0.29 \times 0.29 \times 1.46$ & 16000 & 0.020 & $755 \times 10^{6}$ & 15 & 10,20 & 10,20 \\
N12250 & 5 & $10.23 \times 10.23 \times 14.62$ & $0.29 \times 0.29 \times 1.46$ & 12250 & 0.017 & $967 \times 10^{6}$ & 28 & - & - \\
\hline \hline
\end{tabular}

The time is then sampled with a uniform step of $\Delta t$ satisfying the Courant-Friedrichs-Levy condition: $\Delta t=C h / V_{\max } . V_{\max }$ is the peak phase velocity of a polycrystalline material. $C$ is the Courant number and we have used $C=0.98$ in this work to suppress numerical dispersion while ensuring the stable incremental solution of the elastodynamic problem[47].

The discretized model is then configured to simulate the propagation of plane longitudinal waves, without loss of generality, in the $z$-direction. For this purpose, symmetry boundary conditions (SBCs) are applied to the four outer $x-z$ and $y-z$ surfaces of the cuboid model to constrain their out-of-plane motions. Then, a three-cycle Hann-windowed toneburst signal is applied as a uniformly distributed force in the $z$-direction to all of the nodes on the $x-y$ surface at $z=0$. We note that the initiated wave is quasi-longitudinal[48], whose polarization vector is not perfectly aligned with the wave vector, for wave excitation in an off-symmetry axis direction $\left(\theta_{\mathrm{p}} \neq 0^{\circ}, \theta_{\mathrm{p}} \neq 90^{\circ}\right.$; see Fig. 3(b)) of an elongated polycrystal. However, this misalignment is negligible in our studied cases; see Ref. [38] for details.

The FE models are solved using the elastodynamic time marching FE program Pogo[47,49]. The 
simulation time of the largest FE model of this work, N12250 with about one billion degrees of freedom, amounts to around one hour by using 4× NVIDIA RTX 8000 GPU cards.

During the simulation of a single realization of each FE model, the displacements in the $z$ -direction are monitored across all nodes and at all time steps on the transmitting surface at $z=0$ and on its opposite receiving surface at $z=d_{z}$, forming a through-transmission configuration. As the plane of the equal wave phase is parallel to the transmitting and receiving surfaces, the coherent transmitted and received signals in the time domain, denoted as $U(0 ; t)$ and $U\left(d_{z} ; t\right)$, are obtained by averaging the monitored displacements over all nodes on their respective surfaces at each time step; typical coherent signals are shown in Fig. 4(a). These coherent time-domain signals are then windowed and Fourier transformed into the frequency domain, leading to the signal spectra of $U(0 ; f)$ and $U\left(d_{z} ; f\right)$; typical time windows and amplitude spectra are respectively illustrated in Fig. 4 (a) and (b). Frequency-dependent attenuation is then obtained by comparing the amplitudes of the signals: $\alpha(f)=-\ln \left[A\left(d_{z} ; f\right) / A(0 ; f)\right] / d_{z}$, where $A$ denotes the amplitude part of $U$. The process for determining attenuation (and phase velocity as discussed in Ref. [38]) is the same as in our previous studies[26,27,47], from which more details can be found.

We use the ensemble average of $15 \mathrm{FE}$ simulations (realizations) to obtain statistically converged attenuation results for each modeling case. The multiple realizations are generated by using the same grain structures and re-randomizing the crystallographic orientations of the grains, which was shown to preserve the same statistical variability as the more intense approach of re-randomizing both grain structures and grain orientations[27]. Also, as shown in Table I, each polycrystalline material uses two or three FE material models to accommodate different frequency ranges; some models are simulated with different center frequencies for the transmitted waves. This is adopted to achieve better accuracy and less uncertainty for the FE simulations[47].

The geometric characteristics of polycrystals are represented by the TPC function, which needs to be accurately determined from the FE material models such that it can be incorporated into the theoretical models for direct comparison. The procedures for measuring TPC data from 3D FE 
material models have been previously reported for statistically equiaxed grains[23] and elongated grains[38] so are not repeated here. For the model N11520, the measured TPC statistics for the equiaxed case are given in Fig. 5 as rectangular points while those for the elongated case are shown as circular points for the $\theta_{\mathrm{p}}$ angles of $0^{\circ}, 15^{\circ}, 30^{\circ}, 45^{\circ}$, and $90^{\circ}$. Note that the shown datasets are also representative of the other material models in Table I due to their sufficiently large numbers of grains.
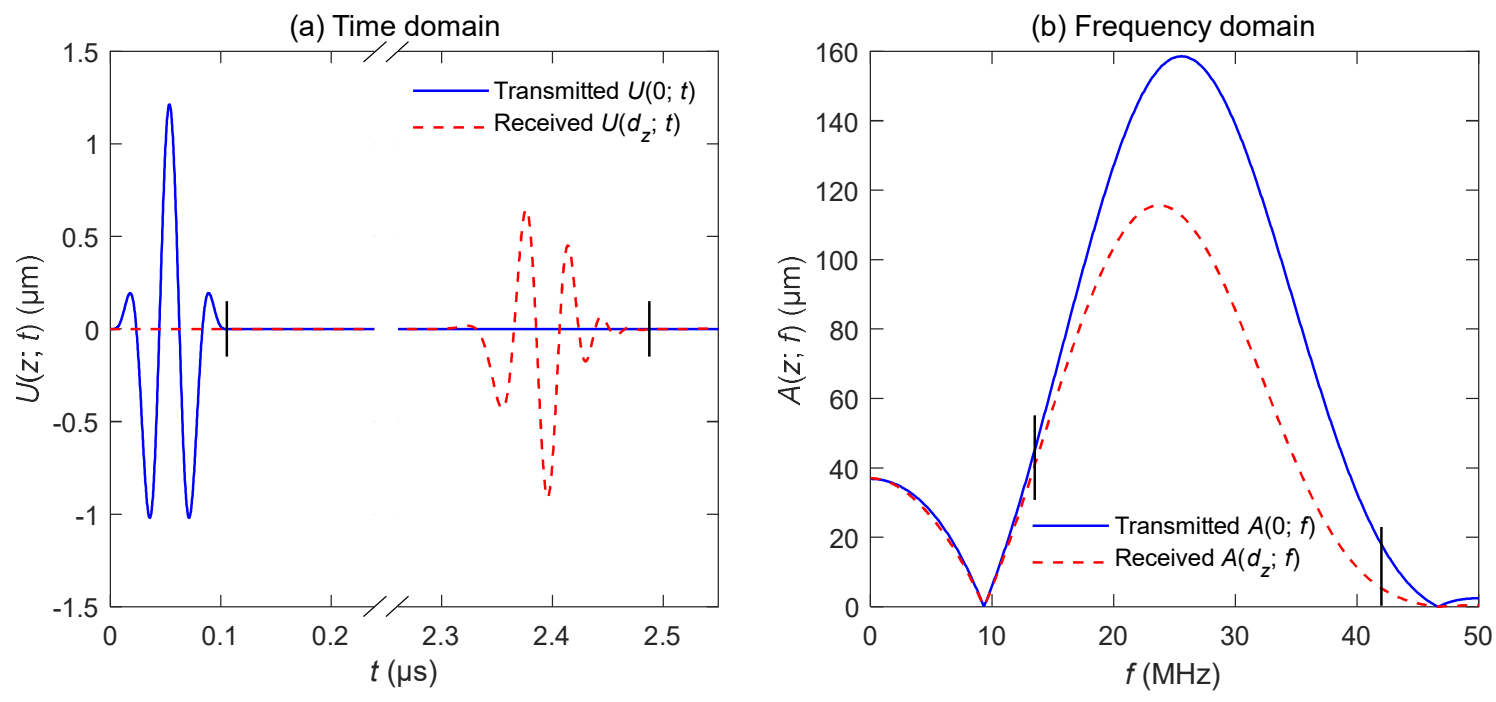

Fig. 4. (Color online) Illustration of coherent (a) time-domain signals and (b) frequency-domain amplitude spectra in the FE simulation of a plane longitudinal wave traveling in aluminum (material properties in Ref. [23]) with statistically elongated grains. The shown simulation uses a single realization of the model N12250 (Table I) and the excitation force is a three-cycle Hann-windowed toneburst with the center frequency of $28 \mathrm{MHz}$. The results in the figure are given for the grain elongated $z$-direction. Each signal in (a) is cropped by using the time window that spans from $t=0$ to the respective vertical mark and then Fourier transformed to get the respective amplitude spectrum in (b). The two vertical marks in (b) show the frequency range where the amplitude spectra are used for attenuation calculation.

Since the statistically elongated grains are scaled from the equiaxed ones, a scaling relation should exist between the measured TPC points of the two cases. Based on the obtained TPC points, we have checked and confirmed that the TPC points of the elongated case indeed have a scaling relation $w\left(r, \theta_{\mathrm{p}}, \varphi_{\mathrm{p}}=0\right)=w\left(r \sqrt{\sin ^{2} \theta_{\mathrm{p}} \cos ^{2} \varphi_{\mathrm{p}} / S_{x}^{2}+\sin ^{2} \theta_{\mathrm{p}} \sin ^{2} \varphi_{\mathrm{p}} / S_{y}^{2}+\cos ^{2} \theta_{\mathrm{p}} / S_{z}^{2}}\right) \quad$ to 
those of the equiaxed case, where the scale factors $S_{i}$ are given in Eq. (15) with the elongation ratio of $R=5$. The root-mean-square (RMS) of the relative difference between the TPC points of the elongated case and those scaled from the equiaxed case is about $0.05 \%$ for all shown angles. This agreement supports the validity of our scaling approach and the assumption of the statistically elongated grains having an ellipsoidal shape on average.

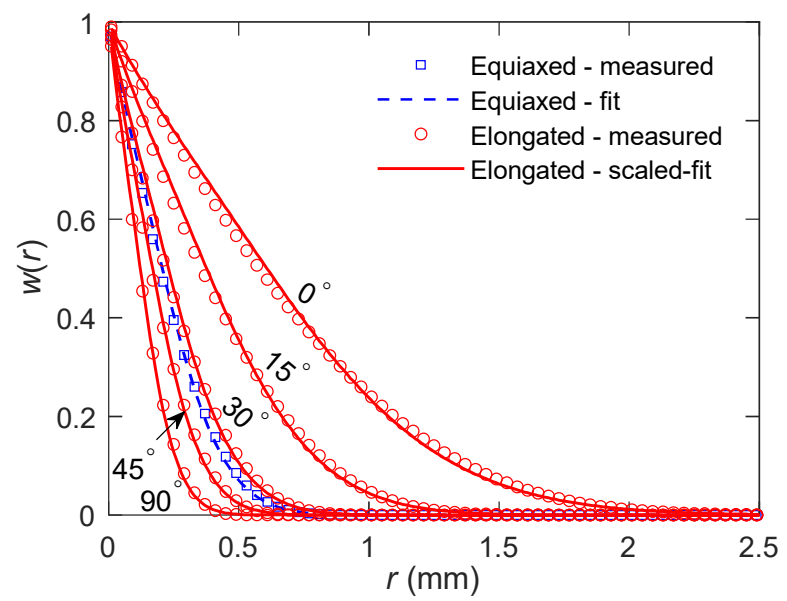

Fig. 5. (Color online) TPC functions of polycrystals with statistically equiaxed and elongated grains. The rectangular and circular points are measured from the model N11520 with statistically equiaxed and elongated grains, respectively. For the elongated case, this work simulates wave propagation at eight different angles of $0^{\circ}, 15^{\circ}, 18.75^{\circ}, 30^{\circ}, 45^{\circ}, 60^{\circ}, 75^{\circ}$, and $90^{\circ}$. For a clean presentation, data points are only shown for $0^{\circ}, 15^{\circ}, 30^{\circ}, 45^{\circ}$, and $90^{\circ}$. The dashed line is the mathematical fit of the rectangular points by the generalized TPC. The solid lines are scaled from the dashed line using the scaling law in Eq. (15). The goodness of the fit, as characterized by the R-square measure, amounts to 0.9992 for all shown cases, indicating a very good agreement between the fit and the respective points. The coefficients of the generalized TPC for both the equiaxed and elongated cases can be found in Table III of Ref. [38].

The measured TPC statistics for the equiaxed case are further represented by a generalized function, $w(r)=\sum_{i} A_{i} \exp \left(-r / a_{i}\right)$, where the best-fit coefficients $A_{i}$ and $a_{i}$ are provided in Table III of our prior work[38]. Although special care should be taken in using this exponential fitting[23,50], the coefficients found for the equiaxed case have ensured a very good agreement in TPC with the measured points and a proper TPC decay outside of the fitting range, as can be seen in Fig. 5. Since the measured TPC points have a scaling relation between the elongated and 
equiaxed cases, the mathematical representation of the TPC function for the elongated case can also be scaled from that of the equiaxed case. This leads to the representation in Eq. (6) for the elongated case, in which the coefficients $A_{i}$ are the same as those of the equiaxed case and the coefficients $a_{x}^{i}, a_{y}^{i}$, and $a_{z}^{i}$ are scaled from those of the equiaxed case $a_{i}$ by using the scale

factors provided in Eq. (15): $\left(a_{x}^{i}, a_{y}^{i}, a_{z}^{i}\right)=a_{i}(1,1, R) / \sqrt[3]{R}$. One should realize that the obtained TPC function represents a smooth surface in 3D space that is fast decaying with distance in any propagation direction. The resulting coefficients can be found in Table III of Ref. [38].

The scaled-fit TPC function is plotted in Fig. 5 as solid lines for all shown angles. The figure demonstrates that the scaled function agrees very well with the measured data points for all angles, indicating again the effectiveness of the scaling approach. We note that a minor deviation is observable between the scaled-fit and the measured points, mostly at $0^{\circ}$ around $r=0.5 \mathrm{~mm}$. This mismatch is brought into the elongated case from the equiaxed case in which a small deviation is present between the fit and the measured points. This is evident since the equiaxed fit and the scaled-fit have the same level of agreement with the respective measurements; the agreement as characterized by the R-square measure amounts to 0.9992 for all shown cases in Fig. 5.

\section{RESULTS AND DISCUSSIONS}

\section{A. Frequency dependencies for the SOA and FE models}

This section presents the FE and theoretical results for the attenuation of longitudinal wave propagation in polycrystals with statistically elongated grains. We aim to evaluate the agreement between the models and to give an insight into the effects of the material systems and grain geometry.

\section{Frequency dependence of attenuation}

Fig. 6 shows normalized attenuation, $2 \alpha_{\mathrm{L}} a_{x}$, versus normalized frequency, $2 k_{0 \mathrm{~L}} a_{x}$. The three panels are given for aluminum, Inconel, and CSP, respectively. $a_{x}=0.22 \mathrm{~mm}$ is the mean grain radius in the shortened $x$-direction as defined by Eq. (11); for reference, the mean grain 
radius in the elongated $z$-direction is $a_{z}=5 a_{x}$. The finite element model (FEM) results are plotted as points in the figure and the SOA predictions are provided as lines. The Rayleigh and stochastic asymptotes derived from the Born approximation of the SOA model are shown as dash-dotted lines. The FEM results are obtained for the material models given in Table I and the average of 15 realizations is used to obtain statistically converged results as presented in the figure; the sizes of the FEM point markers are larger than the standard deviations of the FEM results and the corresponding error bars are thus not shown. The SOA predictions are obtained by using the TPC function of the FE material models as input, and thus the SOA and FEM results are directly comparable.

The SOA and the FEM results approach the Rayleigh asymptote, Eq. (12), at very low frequency and the stochastic asymptote, Eq. (13), at high frequency. The FE attenuation results support a smooth frequency transition from the fourth to the second powers between the Rayleigh and stochastic regimes and the existence of a "hump" in this transition region [11,31]. As in the case for polycrystals with statistically equiaxed grains[11,24,28], this behavior is a result of the change of the dominant scattering mechanism, as the longitudinal-to-transverse wave scattering in the Rayleigh regime is replaced by the longitudinal-to-longitudinal scattering in the stochastic regime. This mode transition is a unique feature for the longitudinal waves. The stochastic regime is not pronounced in the elongated direction for the strongly scattering materials, Inconel and CSP. Relating to iron with statistically equiaxed grains, Stanke and Kino[11] have also noted that the stochastic region becomes shorter as the degree of the grain anisotropy increases.

For Inconel and CSP, the SOA model attenuation displays the geometric regime in the grain elongated direction, where $2 \alpha_{\mathrm{L}} a_{x}$ starts to decrease with frequency. The decline of the attenuation differs from the observation for polycrystals with statistically equiaxed grains by Stanke and Kino[11] and elongated grains by Calvet and Margerin[33], who observed that attenuation is independent of frequency in the geometric regime. We attribute this difference to the difference in the TPC functions. The FEM results have not been obtained at such high frequencies although their highest normalized frequencies are as large as $2 k_{0 \mathrm{~L}} a_{z}=42$ and $2 k_{0 \mathrm{~L}} a_{z}=45$ (this is discussed in detail below). In the frequency range plotted, the results for aluminum does not indicate a departure from the stochastic regime even at $2 k_{0 \mathrm{~L}} a_{z}=70$ for the 
FE and $2 k_{0 \mathrm{~L}} a_{z}=150$ for the SOA models. The geometric region for aluminum occurs at higher frequencies (where $2 \alpha_{\mathrm{L}} a_{x}$ also declines) and it was also observed for statistically equiaxed grains[24].
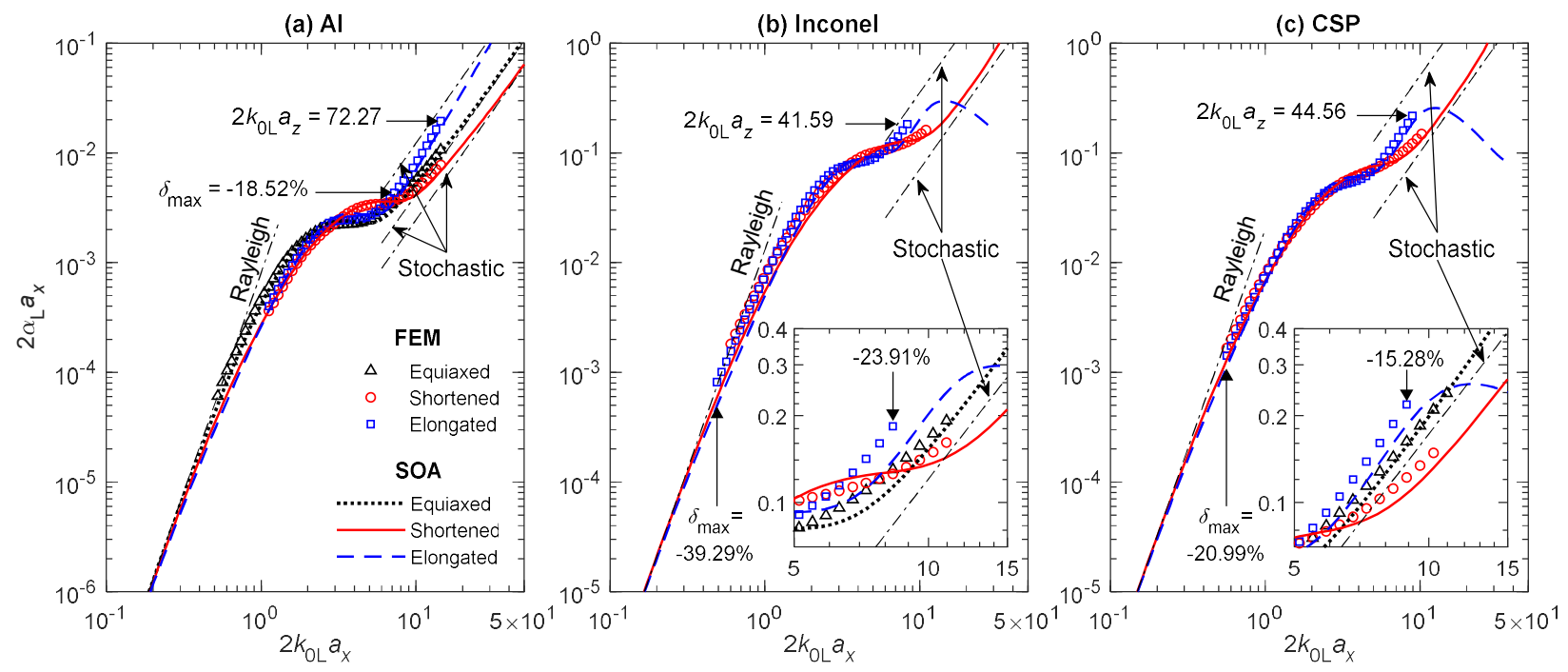

Fig. 6. (Color online) Comparison of normalized longitudinal attenuation between the FEM and SOA models for (a) aluminum, (b) Inconel, and (c) CSP. The results are plotted against normalized frequency $2 k_{0 \mathrm{~L}} a_{x}$, where $a_{x}=0.22 \mathrm{~mm}$ is the mean grain radius in the shortened direction (that of the elongated direction is $a_{z}=5 a_{x}$ ). The dash-dotted lines represent Rayleigh or stochastic asymptotes. $\delta_{\max }$ signifies the maximum difference between the SOA and FEM models, and it occurs in the elongated direction for all three materials. The annotation $2 k_{0 \mathrm{~L}} a_{z}$ specifies the maximum frequency normalized by $a_{z}$ for FE modeling in the elongated direction. For clarity of the plots, only a subset of the calculated FEM points is displayed in the figure and the rest of the points between those displayed are skipped. The figure insets in the Inconel and CSP frames show the high-frequency results for the equiaxed cases (the largest relative difference in the insets between the SOA and FE results is also indicated). At lower frequencies, the equiaxed results are almost indistinguishable from the shown elongated cases. The error bars for the FEM points are not shown since they are smaller than the point markers. All panels use the same legend as given in (a).

The results for statistically equiaxed grains are also provided in Fig. 6 to illustrate the similarities 
and differences between the elongated and equiaxed cases. All results are normalized by the same factor $a_{x}$ as was conventionally used in the study of statistically elongated grains[31]; this factor corresponds to the mean grain radius in the shortened direction, Eq. (11). A different factor can be adopted for the normalization, but it only linearly transforms the data points and curves and does not change the relations between them. Yet, it is important to emphasize that, as annotated in the figure, the FE modeling has reached large normalized frequencies for the elongated direction, when normalized with the mean grain radius $a_{z}$ in the elongated direction.

In the Rayleigh regime, the attenuation of each material is the same in the shortened and elongated directions and equals to the attenuation in the equiaxed grains cases due to attenuation dependence on the effective volume of the grains, which follows from the Rayleigh asymptote Eq. (12). In the high-frequency stochastic regime, grain elongation results in a clear directional effect on the attenuation as it depends on the average grain radius in the direction of wave propagation. While in Fig. 6 we only consider the shortened and elongated directions, we are generally interested in the propagation of waves in directions lying in the plane containing both those directions; the angle dependence will be discussed below in Sec. IV.B.

It is clear from the figure that in general, the SOA and FEM models are in good agreement. The overall difference between the SOA and FE models across the whole frequency range is further quantified by the root-mean-square deviation (RMSD) as normalized by the FEM attenuation; the results for different materials and cases are shown in Table II. The level of agreement for polycrystals with statistically elongated grains is almost the same as that of the equiaxed ones. The RMSD values shown in the table for the equiaxed cases are a bit higher than those in our previous work[24] because higher frequency FEM results are included in the present study. The SOA model in the shortened direction is in very good agreement with the FE model in the whole frequency range for all materials, and the agreement decreases slightly in the elongated direction. This is because the normalized frequency in the elongated direction, $2 k_{0 \mathrm{~L}} a_{z}$, is five times that of the shortened direction, $2 k_{0 \mathrm{~L}} a_{x}$, leading to a higher level of scattering and a larger difference between the SOA and FE models in the elongated direction. The table also shows that the overall difference between the SOA and FE models increases from aluminum through CSP to Inconel, showing a positive correlation with the universal anisotropy factor as already explained in our 
previous study[24]. For the three materials, the maximum difference between the SOA and FE models is marked as $\delta_{\max }$ in the figure and it occurs in the elongated direction for all three materials. For the studied cases the largest relative difference, $-39.29 \%$, is for Inconel at the lowest FE frequency. The discrepancy between the FE and the SOA models is rather modest, considering the approximations involved in both the SOA and FE models. It is important to realize that the difference between the SOA and FE models may also be partially attributed to the symmetry boundary conditions (SBCs) effect in the FE model that is not accounted for in the SOA model, and this SBCs effect may result in a difference of $-20 \%$ for equiaxed Inconel[47].

Table II. RMSD between the SOA and FE models (the FE model is taken as the reference) for different material systems. The universal anisotropy factor[51] $A^{\mathrm{U}}$ and the elastic scattering factors $Q_{\mathrm{L} \rightarrow \mathrm{L}, \mathrm{T}}$ are also provided in the table.

\begin{tabular}{lcccccc}
\hline \hline \multirow{2}{*}{ Materials } & \multirow{2}{*}{$A^{\mathrm{U}}$} & \multirow{2}{*}{$Q_{\mathrm{L} \rightarrow \mathrm{L}}$} & $Q_{\mathrm{L} \rightarrow \mathrm{T}}$ & \multirow{2}{*}{$\begin{array}{c}\text { RMSD for } \\
\text { equiaxed case }\end{array}$} & \multicolumn{2}{c}{ RMSD for elongated case } \\
\cline { 5 - 7 } & & & & & Shortened & Elongated \\
\hline Al & 0.054 & $7.80 \times 10^{-5}$ & $3.34 \times 10^{-4}$ & $10.15 \%$ & $6.81 \%$ & $10.71 \%$ \\
Inconel & 1.420 & $2.26 \times 10^{-3}$ & $7.59 \times 10^{-3}$ & $21.47 \%$ & $20.22 \%$ & $24.77 \%$ \\
CSP & 0.948 & $3.23 \times 10^{-3}$ & $7.19 \times 10^{-3}$ & $10.57 \%$ & $10.47 \%$ & $11.94 \%$ \\
\hline \hline
\end{tabular}

The good agreement between the SOA and FE models indicates that the approximations of the SOA model impose generally acceptable influences, even for polycrystals with relatively high anisotropy grains, such as Inconel. The quality of agreement is all the more surprising considering the key approximations of the SOA model, such as the replacement of the polycrystal by a continuously random medium and accounting only partially for the multiple scattering effects; in the formulations, these representations are assumed to be suitable only under conditions of small wavefield and inhomogeneity perturbations of the medium. Another important assumption in the SOA model is the factoring of the TPC function of a polycrystal into the geometric and elastic parts, Eq. (3), which has been discussed in Sec. II. This factorization has been supported by the direct numerical comparison[42] for the statistically isotropic polycrystals with statistically equiaxed grains; our current and recent[38] study further supports (while indirectly) this factorization for statistically anisotropic polycrystals with elongated grains.

\section{Wavefield fluctuations}


Using the CSP as an example material, Fig. 7 shows the incoherent wavefield fluctuation as normalized by the coherent field in the equal phase plane for different frequencies in both shortened and elongated directions. To obtain the wavefield fluctuation of the propagating field, let us say in the $z$-direction, the $z$-displacements across all points on the receiving plane $z=d_{z}$ are recorded as a spatially varied waveform $u=u(x, y ; t)$. The spectral amplitude field $u=u(x, y ; f)$ is obtained by Fourier transforming the windowed waveform of each point with respect to time. The plotted normalized wavefield fluctuations are $u_{\mathrm{f}}(x, y ; f)=u(x, y ; f) /\langle u(x, y ; f)\rangle_{x, y}-1$, where $\langle\cdot\rangle_{x, y}$ represents the spatial average over all points. A single realization is employed to acquire the fluctuations in the model N11520 for the normalized frequencies of $0.56,1.00$ and 1.39 , and in the model $\mathrm{N} 16000$ for the frequencies of 5.56 and 8.34 .

The RMS of the fluctuations in Fig. 7 increases with frequency till the stochastic regime of the attenuation (Fig. 6) and then the level of fluctuation tends to saturate at high frequencies starting at around $2 k_{0 \mathrm{~L}} a_{x}=5.56$. For the same $2 k_{0 \mathrm{~L}} a_{x}$ and $2 k_{0 \mathrm{~L}} a_{z}$ before the saturation, the fluctuation RMS is higher in the shortened grain direction than in the elongated direction.

As mentioned in Sec. II, the normalized wavefield fluctuations are generally expected to be much smaller than unity in order for the theoretical SOA model to be valid[41]. However, the fluctuations shown in Fig. 7 are surprisingly large for all cases, and the magnitude of fluctuations can be nearly 4 times that of the coherent field. Considering the good agreement between the SOA and FE models in Fig. 6, this suggests that the SOA model works much better than expected under conditions of large wavefield fluctuations.

\section{Upper-frequency limit for the FE calculations}

As discussed in Section IV.A.1 relating to the results in Fig. 6 in the grain elongation direction, the SOA attenuations for Inconel and CSP have reached the geometric region, while the FEM results have not been obtained for frequencies higher than shown (again recall that the highest normalized FE frequencies are as large as $2 k_{0 \mathrm{~L}} a_{z}=42$ for Inconel, $2 k_{0 \mathrm{~L}} a_{z}=45$ for CSP, and $2 k_{0 \mathrm{~L}} a_{z}=70$ for aluminum). This is the upper-frequency limit of the reliable FE simulations in our study for those materials. 

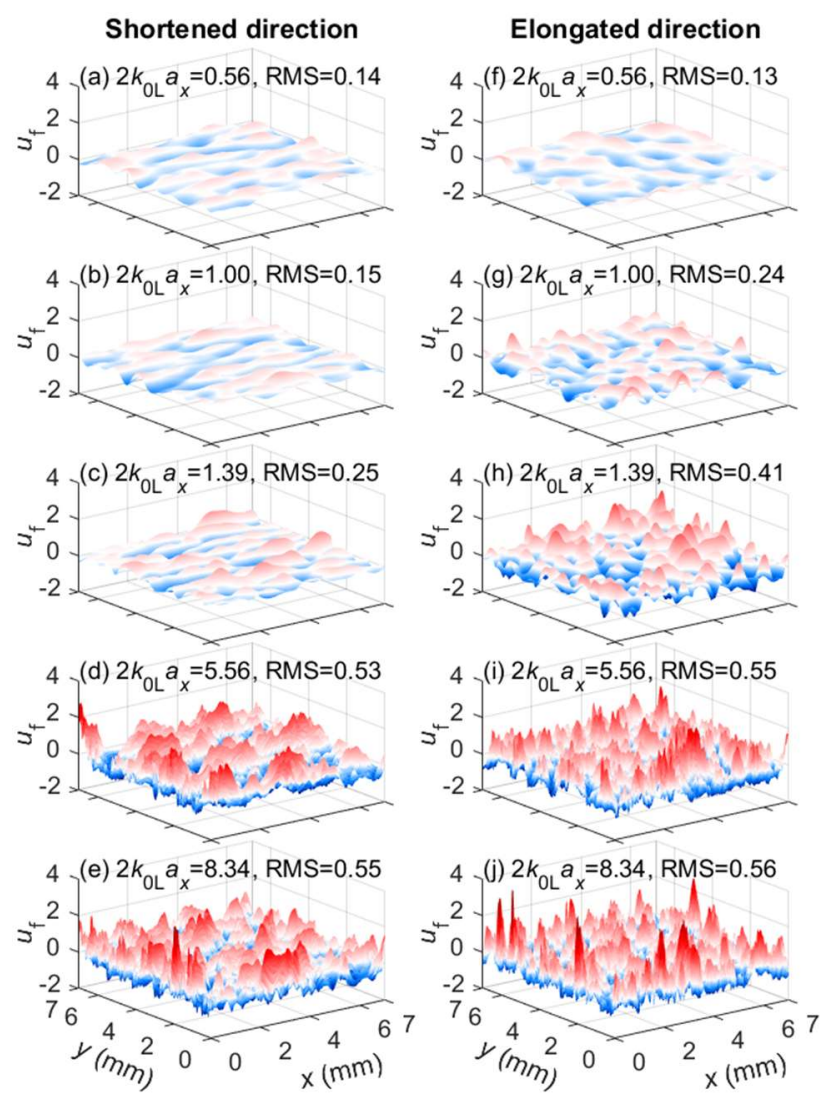

Fig. 7. (Color online) Illustration of wavefield fluctuations for the normalized spectral amplitude field $u_{\mathrm{f}}(x, y ; f)=u(x, y ; f) /\langle u(x, y ; f)\rangle_{x, y}-1$. Panels (a-e) are for shortened and (f-j) for elongated directions at different normalized frequencies. The results are given for the material CSP with statistically elongated grains of elongation ratio 5. The fluctuations at the normalized frequencies of $0.56,1.00$ and 1.39 are acquired from a single realization of the model N11520, and those at 5.56 and 8.34 are obtained from the model N16000.

Further frequency increase has been found to lead to highly distorted time-domain signals and a deteriorated signal-to-noise ratio (SNR). To get a smaller decay and distortion of the coherent signal, we have tried varying the sample length in the wave propagation direction of the FE model; however, this approach has its own limit. One needs to obtain a statistically sufficient number of grains in the propagation direction to represent a statistically meaningful polycrystal volume and to assure the accumulation of a sufficient number of the occurrence of multiscattering events thus achieving a statistically meaningful determination of attenuation. There are on average 10 grains in the elongated direction, which we have accepted as 
sufficient[38], in the high-frequency FE model N16000 (it will be a much smaller number for the largest grains in the propagation direction). For this model, the SNR is represented by the ratio of coherent to incoherent fields, namely the reciprocal of the wavefield fluctuation RMS in Fig. 7, which is about 1.8 at the highest frequency. About the same SNR value is obtained by estimating the noise level recorded in the time domain after the arrival of the coherent signal (the method of computing the signal in the time domain is described in Sec. III; however, the high-frequency FE material model N16000 is used). For the SNR, the highest level of the noise RMS is used in the frequency bandwidth of the coherent signal. Therefore, the highest components of the amplitude spectra are very close to the noise level. The same conclusion can be made for the wave propagation perpendicular to the grain elongation direction of the polycrystal since the RMS of wavefield fluctuations is about the same at the highest frequencies, Fig. 7. If we include higher frequencies and noisier parts of the spectrum in the attenuation analysis, the dependence of the attenuation on the propagation distance appears at those frequencies (such behavior was actually observed in prior experimental work[2]). For these reasons, in this and our recent work [38], we have limited our FE frequency range to that shown in Fig. 6. The attempt to further increase the high-frequency range is beyond the scope of this study and will be explored in future work.

The FEM points shown in Fig. 6 and in the results given below are statistically converged and have a high degree of numerical accuracy[47]. In the elongated direction of Inconel and CSP, when the SOA model begins transition to the geometric region, it seems that the highest frequency FEM points (insets in Fig. 6) deviate from the SOA model and continue to grow with frequency. Here we have to emphasize that the results for the transition to, and in, the geometric region as described by the SOA model may also not be valid for polycrystals with high anisotropy grains and thus large material inhomogeneity perturbations[32]. Bourret[52] has considered the iteration-series solution applied to the time-dependent system which has an exact solution. The diagrammatic method he used is similar to that employed for his analysis of wavefields in random media[40]. It was found[52] that different perturbation solutions diverge from each other and the exact solution with the increase of perturbation strength. Also, the condition of the smallness of the generalized Reynolds number[15] is not satisfied at high frequencies. Therefore, some discrepancy between the SOA and the FE results at the highest points of the FE simulation, while acceptable, still require better understanding. This may be addressed in future studies by improving the signal-to-noise ratio of the FE simulations in the 
higher frequency range.

\section{B. Effect of grain shape on attenuation}

\section{Directional ratio}

We have mentioned above that the grain elongation causes the directional dependence of attenuation at high frequencies. Here we use the ratio of attenuation between the elongated and shortened directions as a measure to quantify this. The elastic part of attenuation nearly cancels out after division in the ratio, and thus the effect of the geometric part on attenuation can be observed clearly. The ratios are plotted in Fig. 8 for the three materials. As with the original attenuation results shown in Fig. 6, the SOA ratios (lines) agree very well with the FEM ratios (points) across the whole frequency range.

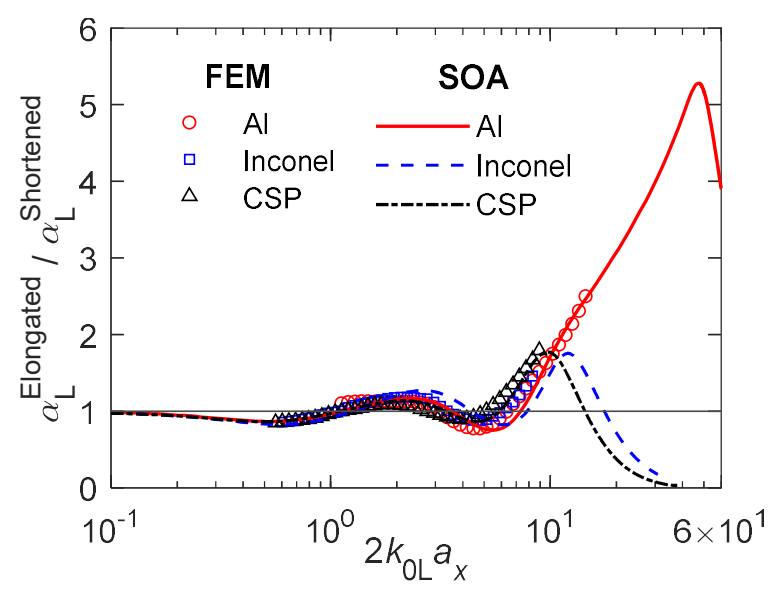

Fig. 8. (Color online) Directional ratio of longitudinal attenuation between the elongated and shortened directions. The horizontal reference line shows the ratio of unity. For clarity, only a subset of the original FEM points is displayed in the figure and the remaining points between those displayed are skipped.

The attenuation ratios overlap reasonably well for all materials and for both the FE and SOA models except above the stochastic region when the transition to the geometric region begins; here each ratio reaches a separate peak and then begins to decline. This behavior of a peak followed by decline was also discussed in Rokhlin et al.[16]. As discussed above in Sec. IV.A, the abrupt drop is due to the emergence of the geometric regime in the elongated direction. For Inconel and CSP, the ratio only reaches the value of about 2, which is smaller than the grain 
elongation ratio of 5 . However, for aluminum, which has a relatively weak scattering, the peak occurs much later in frequency and the attenuation ratio is 5.28 , which is a bit greater than the grain elongation ratio. The FE computations did not reach those frequencies.

In the low-frequency Rayleigh regime, the ratio is almost unity, confirming that attenuation is independent of the grain shape. The Rayleigh-stochastic transition exhibits itself as the non-monotonic variations that are caused by the transition of dominant scattering mechanisms between the two regimes. Consistently for all three materials, the ratio first decreases below one (scattering in a shorter direction is a bit stronger) when departing from the Rayleigh region. This occurs relatively early in frequency and is supported by the FEM simulations. Importantly, for all studied materials, the directional attenuation ratio is about one until the normalized frequency $2 k_{0 \mathrm{~L}} a_{x}$ increases to five for the SOA and FE models.

\section{Polar dependencies}

The evident directional difference might be useful for inversely inferring the average shape of the elongated grains. To further elucidate the directional behavior we seek to evaluate the change of attenuation with propagation angle $\theta_{\mathrm{p}}$, which denotes the angle between the directions of propagation and grain elongation as illustrated in Fig. 1 and Fig. 3(b). We use CSP as the example material for this evaluation and simulate the propagation of waves in the directions of $0^{\circ}$ (elongated direction), $15^{\circ}, 18.75^{\circ}, 30^{\circ}, 45^{\circ}, 60^{\circ}, 75^{\circ}$, and $90^{\circ}$ (shortened direction). The FEM results together with the predictions of the SOA model are shown in Fig. 9 versus the normalized frequency for the propagation directions of $0^{\circ}, 15^{\circ}, 18.75^{\circ}, 30^{\circ}, 45^{\circ}$, and $90^{\circ}$; the results for $60^{\circ}$ and $75^{\circ}$ are not shown as they complicate the visualization but they will be used later in Fig. 10. The same modeling conditions (model dimensions, mesh size, center frequency, etc.) are used to perform the FE simulations for different propagation directions, but the FE results are given for different frequency ranges in order to have the same level of numerical accuracy[47].

The agreement between the FEM and SOA results is generally good for all plotted directions. The largest difference occurs in the elongated direction $\left(0^{\circ}\right)$ because the FEM result continues to grow while the SOA model is transiting into the geometric regime where attenuation decreases. As the angle deviates from the elongated direction, the mean grain radius in the propagation direction decreases, and therefore in the stochastic region the level of attenuation should also 
decrease as follows from the Born approximation[17,31-34] and the stochastic limit of the SOA model, Eq. (13). This is valid for the angles of $30^{\circ}, 45^{\circ}$, and $90^{\circ}$ closer to the shortened direction which is supported by both the FEM and SOA models. However, it is not the case as attenuation approaches the transition to the geometric region which is observed for the angles in the vicinity of the elongated direction. At those angles $\left(0^{\circ}, 15^{\circ}\right.$, and $\left.18.75^{\circ}\right)$, the FEM results nearly overlap, while the SOA attenuation for $0^{\circ}$ is even smaller than those for $15^{\circ}$ and $18.75^{\circ}$ since it is deeper into transition to the geometric region. The SOA model results in Fig. 9 are in general reminiscent of those in Fig. 6 of Yang et al.[32] that were obtained for ellipsoidal scatterers in the scalar approximation; however, near-zero propagating angles were not investigated in that work.

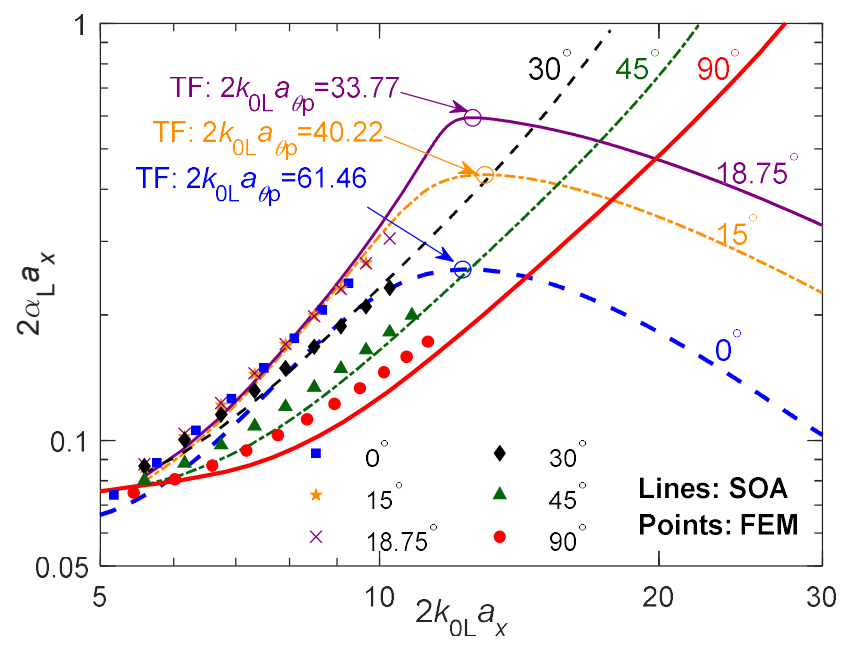

Fig. 9. (Color online) Normalized longitudinal attenuation versus normalized frequency in different propagation directions for triclinic CSP; points are for the FEM results and lines are for the SOA (The propagation directions $\theta_{\mathrm{p}}$ are $0^{\circ}, 15^{\circ}, 18.75^{\circ}, 30^{\circ}, 45^{\circ}$, and $90^{\circ}$ ). The FEM points for $0^{\circ}$ (squares), $15^{\circ}$ (stars), and $18.75^{\circ}$ (crosses) are mostly overlapped in the given frequency range. TF denotes the normalized transition frequency $2 k_{0 L} a_{\theta \mathrm{p}}$ to the geometric regime, and it corresponds to the peak of the SOA curve. The normalization factor $a_{\theta \mathrm{p}}$ for TF is the mean grain radius in the wave propagation direction $\theta_{\mathrm{p}}$. For clarity, only a subset of the original FEM points is displayed in the figure and the remaining points between those displayed are skipped.

It is shown in Fig. 9 that at the angles $0^{\circ}, 15^{\circ}$, and $18.75^{\circ}$ the $\mathrm{SOA}$ attenuation transits into the 
geometric regime at different $2 k_{0 \mathrm{~L}} a_{\theta \mathrm{p}}$ (as indicated in the figure by the TF points, open circles) and roughly the same nondimensional frequency $2 k_{0 \mathrm{~L}} a_{x}$; the transitions occur later in frequency for the propagation at larger angles $30^{\circ}, 45^{\circ}$, and $90^{\circ}$ and they are not shown in the figure. The normalization factor $a_{\phi p}$ for the TF is the mean grain radius in the wave propagation direction $\theta_{\mathrm{p}}$ as defined by Eq. (10). As for the $0^{\circ}$ and $90^{\circ}$ degrees, the FEM results are not obtained at such high frequencies (Sec. IV.A.3) and available only below the transition to the geometric region.

The directional dependence is further evaluated in Fig. 10 by polar plotting the normalized attenuation against propagation direction $\theta_{\mathrm{p}}$ for the normalized frequencies of 5.56 and 8.34. The FEM results are mirrored about the shortened and elongated axes to obtain all FEM points as plotted in the figure. The SOA and Born approximation results are also provided in the figure as solid and dash lines, respectively. The Born approximation model is included here because prior studies are mostly based on this model[17,32].

The panels (c-d) of Fig. 10 show for comparison the stochastic asymptote (dash-dotted line) that has the same elliptical shape as the mean elongated grain with the aspect ratio of 5 . This is consistent with the accepted view $[31,33,34]$ that the stochastic asymptote follows the same shape as the statistical geometry of the grains. The difference with the FEM results indicates again that for the CSP material the stochastic regime did not develop.

Consistent with Fig. 9, the SOA and Born approximation models in general match very well with the FE model. The SOA model exhibits a more complicated behavior at, and near, the elongation direction, $0^{\circ}$. The relative differences between the SOA and FE models are given in Table III for the plotted frequencies and simulated angles. The largest deviations from the FEM are at a near-zero angle. As shown in Fig. 9, at the higher frequency of 8.34, the largest attenuation is found in both the SOA and FE models in the vicinity of the propagation direction $18.75^{\circ}$. The complexity of the behavior arises because the different propagation angles are associated with different scattering regimes of the SOA model involving the stochastic, stochastic-geometric transition, or geometric regimes. For example, in the elongated direction $\left(0^{\circ}\right)$ that has the largest difference between the SOA and FE models, the SOA model is already transiting into the 
geometric region while the FEM result is still increasing with frequency as shown in Fig. 9. The Born approximation does not exhibit this nonmonotonic behavior with angle since it does not follow the transition into the geometric region as was shown in Fig. 2.

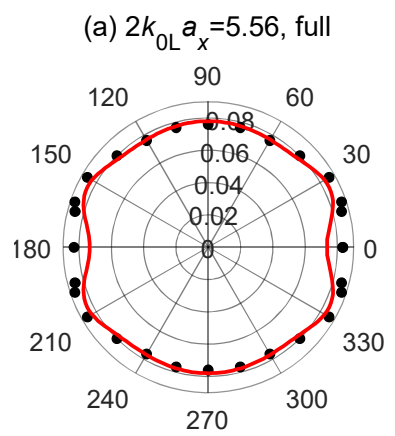

(c) $2 k_{0 \mathrm{~L}} a_{x}=5.56$, quarter
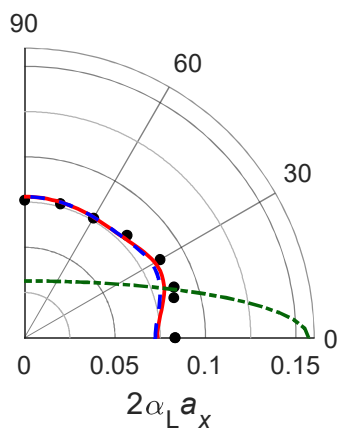

(b) $2 k_{0 \mathrm{~L}} a_{x}=8.34$, full

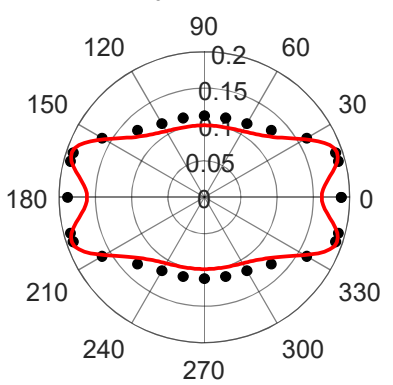

(d) $2 k_{0 \mathrm{~L}}{ }^{a}=8.34$, quarter

90

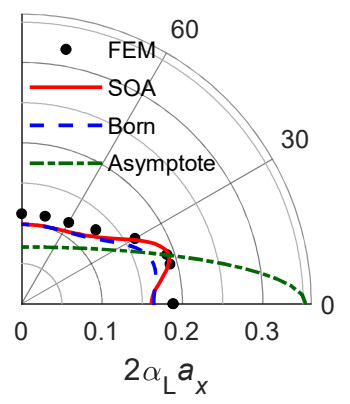

Fig. 10. (Color online) Polar plot of normalized longitudinal attenuation ( $r$-axis) versus wave propagation direction ( $\theta$-axis) for CSP at the normalized frequencies $2 k_{0 \mathrm{~L}} a_{x}$ of (a) 5.56 and (b) 8.34. (c-d) show the respective quarter plots of (a-b) with the Born approximation and asymptote curves added. Points are for the FEM results and solid lines are for the SOA predictions; dash and dash-dotted lines represent respectively the Born approximation and the elliptical stochastic asymptotes. All panels use the same legend as shown in (d).

Table III. Relative difference of longitudinal attenuation between the SOA and FE models for polycrystalline CSP with statistically elongated grains.

\begin{tabular}{lrrrrrrrr}
\hline \hline \multirow{2}{*}{$2 k_{0 \mathrm{~L}} a_{x}$} & \multicolumn{8}{c}{$\delta=\alpha_{\mathrm{L}}^{\mathrm{SOA}} / \alpha_{\mathrm{L}}^{\mathrm{FEM}}-1(\%)$} \\
\cline { 2 - 9 } & $0^{\circ}$ & $15^{\circ}$ & $18.75^{\circ}$ & $30^{\circ}$ & $45^{\circ}$ & $60^{\circ}$ & $75^{\circ}$ & $90^{\circ}$ \\
\hline 5.56 & -11.60 & -7.05 & -6.52 & -3.74 & -2.62 & 0.61 & 1.18 & 2.58 \\
8.34 & -14.25 & -1.02 & 1.05 & -2.08 & -8.44 & -10.18 & -11.33 & -11.69 \\
\hline \hline
\end{tabular}




\section{Effect of elastic scattering factors on attenuation}

Now we proceed to evaluate the dependence of attenuation on the elastic properties of the polycrystals. The FEM and SOA results of all three materials given in Fig. 6 are replotted in Fig. 11(a) and (c) for the shortened and elongated directions, respectively. The curves for the CSP and Inconel are nearly overlapped, this is because the $Q_{\mathrm{L} \rightarrow \mathrm{T}}$ and $Q_{\mathrm{L} \rightarrow \mathrm{L}}$ factors are relatively close (Table II). However, the attenuation for aluminum is much smaller. By comparing the results of the three materials, we can intuitively evaluate the impact of elastic properties on attenuation. However, the evaluation is made more quantitative in panels (b) and (d) by scaling the attenuation by the elastic factors that affect the attenuation of the low-frequency Rayleigh and high-frequency stochastic regimes. We emphasize that both the FEM points and SOA lines are equally scaled and the resulting overlap across the different material systems supports the universality of the scaling parameters.

In the low-frequency Rayleigh regime, the attenuation results are divided by the elastic factor $Q_{\mathrm{L} \rightarrow \mathrm{T}} V_{0 \mathrm{~L}}^{3} / V_{0 \mathrm{~T}}^{3}$. This factor is chosen based on Eq. (12) and the factor $Q_{\mathrm{LL}}^{*}$ in the equation is neglected because it is small compared to the chosen factor. Panels (b) and (d) show that after division the results for different materials nearly overlap for both the SOA and FE models and both the shortened and elongated directions. The overlap means that the proportionality of attenuation to the chosen elastic factor holds for the Rayleigh regime. The studied materials have similar velocity ratios as their Poisson's ratios are close, and thus the factor being divided can be substituted by $Q_{\mathrm{L} \rightarrow \mathrm{T}}$ with small changes for the overlap. As a result, the mode-converted elastic

scattering factor, $Q_{\mathrm{L} \rightarrow \mathrm{T}}$, is usually used to describe the degree of inhomogeneity perturbation of polycrystals in the long-wavelength scattering range[16,24].

In the high-frequency stochastic regime, the attenuation results are divided by the elastic scattering factor $Q_{\mathrm{L} \rightarrow \mathrm{L}}$, which is similarly chosen based on Eq. (13). It is clear from panels (b) and (d) that the FEM points and SOA curves overlap among different materials. In panel (b), the results for CSP slightly deviate from those of the other two materials below the normalized frequency of 15 , which is because CSP reaches the stochastic regime later than the other two materials. The overall good overlap of the three materials suggests that the attenuation in the 
stochastic regime is elastically determined by the factor $Q_{\mathrm{L} \rightarrow \mathrm{L}}$. This conclusion is important because it means that the level of inhomogeneity perturbation of polycrystals can be solely quantified by the elastic scattering factor $Q_{\mathrm{L} \rightarrow \mathrm{L}}$ in the short wavelength scattering range[16,24].
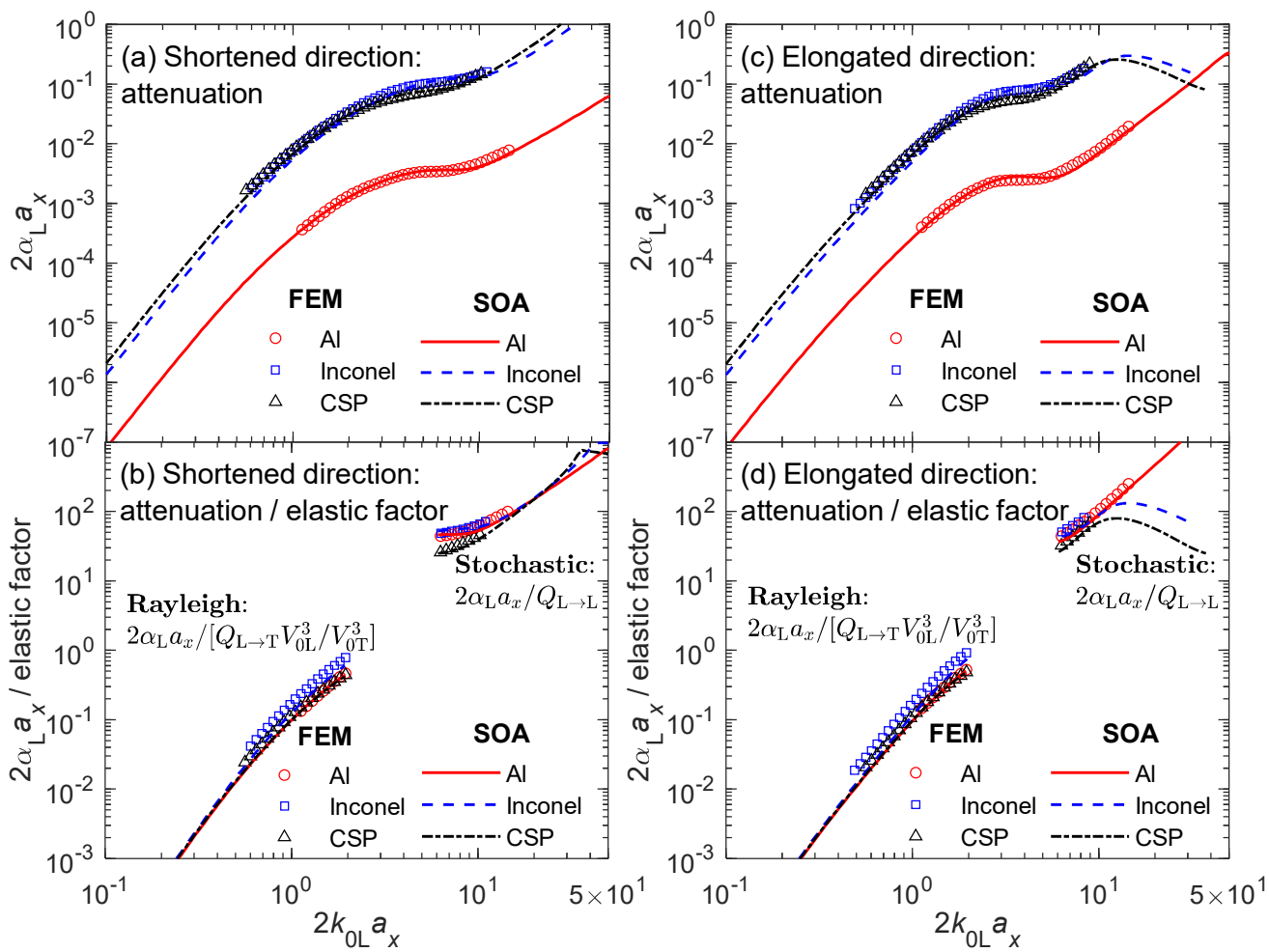

Fig. 11. (Color online) Comparison of normalized longitudinal attenuation between the three polycrystalline material systems in the (a) shortened and (c) elongated directions. (b) and (d) show the respective results of (a) and (c) divided by the elastic factors. The elastic factor being divided is $Q_{\mathrm{L} \rightarrow \mathrm{T}} V_{0 \mathrm{~L}}^{3} / V_{0 \mathrm{~T}}^{3}$ for the low-frequency range $\left(2 k_{0 \mathrm{~L}} a_{x} \leq 2\right)$, while the factor is $Q_{\mathrm{L} \rightarrow \mathrm{L}}$ for the high-frequency range ( $2 k_{0 \mathrm{~L}} a_{x} \geq 6$ ). For clarity, only a subset of the original FEM points is displayed in the figure and the remaining points between those displayed are skipped.

The scaling results show that the two scattering factors are needed to normalize the level of inhomogeneity perturbation for the Rayleigh and stochastic regimes. These two factors are sufficient to describe the grain elastic property effect on the attenuation in the whole frequency range. The attenuation scaling for different material systems was also discussed in Ref [16]; for the transverse wave, the attenuation scaling can be achieved by a single elastic scattering parameter[37]. A scaling in frequency by a single inhomogeneity factor $\varepsilon^{2}$ to normalize the 
attenuation curves in the stochastic regime was used by Stanke and Kino[11] (for longitudinal waves, the factor they used is the same as our elastic scattering factor $Q_{\mathrm{L} \rightarrow \mathrm{L}}$ ).

\section{SUMMARY AND CONCLUSIONS}

Two models, a theoretical second-order approximation (SOA) model and a numerical three-dimensional (3D) finite element (FE) model, are developed for elastic wave propagation in untextured single-phase polycrystals with statistically elongated grains of arbitrary crystal symmetry. Direction and frequency-dependent scattering-induced attenuation for longitudinal waves is compared for those two models and its relation to frequency, grain elongation and elastic scattering factor are evaluated.

The developed SOA attenuation model for longitudinal and shear propagating waves in statistically elongated polycrystals accounts for some degree of multiple scattering and is applicable in the whole frequency range. The Born approximation and the analytical Rayleigh and stochastic asymptotes are obtained from the SOA model. The SOA model incorporates the accurate geometric two-point correlation (TPC) function sampled from the FE material model, enabling the direct comparison of the two models.

The 3D FE model is implemented for longitudinal waves and for polycrystals with average grain shape being ellipsoid of revolution. Each realization represents material volume comprising specific randomly shaped grains that are contiguous without gaps or overlaps. It simulates wave

propagation in realistic sample volumes of a significant number of grains, and it considers the contribution of all possible multiple scattering events. Three polycrystalline materials, aluminum, Inconel, and the triclinic CSP, are simulated with a grain elongation ratio of 5.

Based on the comparative studies of the SOA and FE models, the following physical conclusions are reached:

1) Quantitative agreement has been found between the two models. The normalized difference between the SOA and FE models is quantified for the attenuation by the RMSD, Table II. In general, the RMSD increases with the universal anisotropy factor $A^{\mathrm{U}}$ and the elastic scattering factors, the parameters which are related to the level of scattering. The RMSD increases from about $11 \%$ for aluminum through $12 \%$ for CSP to $25 \%$ for Inconel, and it is a bit larger for the 
elongated direction than for the shortened direction for all studied materials. The largest point-by-point difference of attenuation between the SOA and the FE models is observed for Inconel, which has the highest inhomogeneity perturbation parameter; the relative difference value is $-39 \%$, see Fig. 6 .

2) The analytical SOA model has a very good agreement with the $3 \mathrm{D}$ FE simulations for attenuation even when the wavefield fluctuations are surprisingly large, Fig. 7. For the CSP, the magnitude of fluctuations in the stochastic regime may be nearly 4 times that of the displacement mean (the coherent part of the total field); at this extreme case, the relative difference between the SOA and FE models is at a reasonable level of around $-15 \%$.

3) The good agreement between the SOA and FE models indicates that the approximations of the SOA model impose generally only small effects in the frequency range studied, even for polycrystals with relatively high anisotropy grains, such as Inconel. Considering the key approximations of the SOA model, such as the replacement of the discrete polycrystals by a continuously random medium, and accounting only partially for the multiple scattering effects, the agreement is remarkable. Another important assumption in the SOA model is the factorization of the TPC function of a polycrystal into the geometric and elastic parts, Eq. (3). This factorization has been supported by the direct numerical comparison[42] for the statistically isotropic polycrystals with equiaxed grains; our study further supports (while indirectly) this factorization for statistically anisotropic polycrystals with elongated grains.

4) Both the SOA and the FE models show that in the Rayleigh regime the attenuation is independent of grain shape and is proportional to the effective volume of the grains and the fourth-degree of frequency in the Rayleigh regime. The attenuation shows a complex dependence on grain elongation and frequency in the Rayleigh-stochastic transition. As scattering enters the stochastic regime, attenuation exhibits a direction dependence and is proportional to the frequency squared; for the strongly scattering materials Inconel and CSP, the stochastic regime is not pronounced in the elongated direction. The SOA model shows that as a result of grain elongation, the onset of the geometric regime varies with the propagation direction and the dependence of the attenuation on direction becomes complicated.

5) In addition to the effect of grain elongation and frequency, the elastic properties of the 
polycrystals also impose a major influence on attenuation. This manifests itself mostly through the two elastic scattering factors, $Q_{\mathrm{L} \rightarrow \mathrm{T}}$ and $Q_{\mathrm{L} \rightarrow \mathrm{L}}$, that are combinations of crystallite elastic constants. In the Rayleigh regime the attenuation coefficient is proportional to the $Q_{\mathrm{L} \rightarrow \mathrm{T}}$ and in the stochastic regime to the $Q_{\mathrm{L} \rightarrow \mathrm{L}}$ factor. Both the FE and SOA results show the scalability of the attenuation by those factors.

\section{ACKNOWLEDGEMENTS}

The authors acknowledge Dr. Anton Van Pamel for his valuable discussions on the FE modeling of elongated polycrystals. M.H. was supported by the Chinese Scholarship Council and the Beijing Institute of Aeronautical Materials. P.H. was funded by the UK Engineering and Physical Sciences Research Council (EPSRC) fellowship EP/M020207/1. M.J.S.L. was partially sponsored by the EPSRC. G.S. and S.I.R. were partially supported by the AFRL (USA) under the prime contract FA8650-10-D-5210.

\section{APPENDIX A. INNER PRODUCT COEFFICIENTS AND ELASTIC SCATTERING FACTORS}

This appendix provides a summary of the inner product coefficients and elastic scattering factors for macroscopically isotropic (untextured) polycrystalline materials of any crystallite symmetry; readers are referred to Sha et al.[24] for expressions and more details.

Ten of the inner product coefficients $A_{M N}$ are given as $[16,24]$

$$
\begin{aligned}
& A_{\mathrm{LL}}=\left\langle\delta c_{13} \delta c_{13}\right\rangle \\
& B_{\mathrm{LL}}=-2\left\langle\delta c_{13} \delta c_{13}\right\rangle+4\left\langle\delta c_{15} \delta c_{15}\right\rangle+2\left\langle\delta c_{13} \delta c_{33}\right\rangle \\
& C_{\mathrm{LL}}=\left\langle\delta c_{33} \delta c_{33}\right\rangle+\left\langle\delta c_{13} \delta c_{13}\right\rangle-4\left\langle\delta c_{15} \delta c_{15}\right\rangle-2\left\langle\delta c_{13} \delta c_{33}\right\rangle \\
& A_{\mathrm{LT}}=\left\langle\delta c_{14} \delta c_{14}\right\rangle+\left\langle\delta c_{15} \delta c_{15}\right\rangle \\
& B_{\mathrm{LT}}=\left\langle\delta c_{15} \delta c_{15}\right\rangle-\left\langle\delta c_{14} \delta c_{14}\right\rangle+C_{\mathrm{LL}} \\
& C_{\mathrm{LT}}=C_{\mathrm{TL}}=-C_{\mathrm{TT}}=-C_{\mathrm{LL}}, A_{\mathrm{TL}}=A_{\mathrm{LT}}, B_{\mathrm{TL}}=B_{\mathrm{LT}}
\end{aligned}
$$

while the remaining two coefficients $A_{\mathrm{TT}}$ and $B_{\mathrm{TT}}$ are provided in Sha and Rokhlin[37]. $\left\langle\delta c_{i j} \delta c_{k l}\right\rangle$ represents the assemble average of the product between $\delta c_{i j}$ and $\delta c_{k l}$, and it can be 
further expressed as a quadratic function of the elastic constants[16,24]. $\delta c_{i j}$ is the spatial fluctuation of elastic tensor with respect to the ensemble average $\left\langle c_{i j}\right\rangle: \delta c_{i j}=c_{i j}-\left\langle c_{i j}\right\rangle$. The ensemble average $\left\langle c_{i j}\right\rangle$ is also widely known as the Voigt average and it relates to the Voigt longitudinal and transverse wave speed via $V_{0 \mathrm{~L}}=\sqrt{\left\langle c_{11}\right\rangle / \rho}$ and $V_{0 \mathrm{~T}}=\sqrt{\left\langle c_{44}\right\rangle / \rho}$, respectively.

The elastic scattering factors $Q_{M \rightarrow N}$ are expressed as[16,24]

$$
Q_{M \rightarrow N}=\left\{\begin{array}{cc}
\frac{A_{M M}+B_{M M}+C_{M M}}{4 \eta \rho^{2} V_{0 M}^{4}} & M=N \\
\frac{A_{M N}+B_{M N} / 3+C_{M N} / 5}{4 \eta \rho^{2} V_{0 M}^{2} V_{0 N}^{2}} & M \neq N
\end{array}\right.
$$

where the factor $\eta$ takes the value of 1 and 2 for longitudinal $(M=\mathrm{L})$ and $\operatorname{transverse}(M=\mathrm{T})$ propagating waves, respectively. The elastic factor $Q_{M M}^{*}=\left(A_{M M}+B_{M M} / 3+C_{M M} / 5\right) /\left(4 \eta \rho^{2} V_{0 M}^{4}\right)$ has also been introduced in this work to simplify the notation of the equations; note that it differs from the elastic scattering factors $Q_{M \rightarrow N}$ as defined in (A-2).

\section{APPENDIX B. DERIVATION OF THE RAYLEIGH ATTENUATION ASYMPTOTE}

The Rayleigh asymptote for attenuation is derived based on the Born approximation. At the low-frequency Rayleigh limit, the wavelength is large compared to the average grain radius. Thus, the TPC function in Eq. (5) can be simplified to $W_{M \rightarrow N}(k, \omega, \xi, \theta, \varphi)=\sum_{i=1}^{n}\left(A_{i} a_{x}^{i} a_{y}^{i} a_{z}^{i}\right) / \pi^{2}$ by neglecting the dimensionless wave number $q_{x}^{2}\left(a_{x}^{i}\right)^{2}+q_{y}^{2}\left(a_{y}^{i}\right)^{2}+q_{z}^{2}\left(a_{z}^{i}\right)^{2}$. For the attenuation component $\alpha_{M \rightarrow N}$ in Eq. (9), the TPC function can now be pulled out of its double integral and the remaining integral can be obtained as $4 \pi\left(A_{M N}+B_{M N} / 3+C_{M N} / 5\right)$, leading to

$$
\alpha_{M \rightarrow N}=\frac{\omega^{4} \sum_{i=1}^{n}\left(A_{i} a_{x}^{i} a_{y}^{i} a_{z}^{i}\right)\left(A_{M N}+B_{M N} / 3+C_{M N} / 5\right)}{\eta \rho^{2} V_{0 M}^{3} V_{0 N}^{5}}
$$

By summing up the two components $\alpha_{M \rightarrow M}$ and $\alpha_{M \rightarrow N}$, the total attenuation for wave $M$ in 
the low-frequency Rayleigh limit can be derived as

$$
\alpha_{M}^{\mathrm{R}}=\frac{1}{2 \pi} k_{0 M}^{4} V_{\mathrm{eff}}^{\mathrm{g}}\left(Q_{M M}^{*}+\frac{V_{0 M}^{3}}{V_{0 N}^{3}} Q_{M \rightarrow N}\right)
$$

where $M, N \in\{\mathrm{L}, \mathrm{T}\}$ and $M \neq N . V_{\mathrm{eff}}^{\mathrm{g}}=8 \pi \sum_{i} A_{i} a_{x}^{i} a_{y}^{i} a_{z}^{i}$ is the effective grain volume. The mode-converted elastic scattering factor $Q_{M \rightarrow N}$ and the elastic factor $Q_{M M}^{*}$ are given in Appendix A.

\section{REFERENCES}

${ }^{1}$ R. B. Thompson, "Elastic-wave propagation in random polycrystals: fundamentals and application to nondestructive evaluation," in Topics in Applied Physics. Imaging of complex media with acoustic and seismic waves, Vol. 84, edited by M. Fink, W. A. Kuperman, J.-P. Montagner, and A. Tourin (Springer-Verlag, Berlin, Germany, 2002), pp. 233-257.

${ }^{2}$ R. B. Thompson, F. J. Margetan, P. Haldipur, L. Yu, A. Li, P. D. Panetta, and H. Wasan, "Scattering of elastic waves in simple and complex polycrystals," Wave Motion 45, 655-674 (2008).

${ }^{3}$ S. Shahjahan, A. Aubry, F. Rupin, B. Chassignole, and A. Derode, "A random matrix approach to detect defects in a strongly scattering polycrystal: How the memory effect can help overcome multiple scattering," Appl. Phys. Lett. 104, (2014).

${ }^{4}$ A. Van Pamel, C. R. Brett, and M. J. S. Lowe, "A methodology for evaluating detection performance of ultrasonic array imaging algorithms for coarse-grained materials," IEEE Trans. Ultrason. Ferroelectr. Freq. Control 61, 2042-2053 (2014).

${ }^{5}$ Y. Liu, C. Liu, A. Van Pamel, and P. Cawley, "Feasibility and Reliability of Grain Noise Suppression in Monitoring of Highly Scattering Materials," J. Nondestruct. Eval. 36, 1-13 (2017).

${ }^{6}$ O. I. Lobkis and S. I. Rokhlin, "Characterization of polycrystals with elongated duplex microstructure by inversion of ultrasonic backscattering data," Appl. Phys. Lett. 96, 161905 (2010).

${ }^{7}$ X. Li, X. Han, A. P. Arguelles, Y. Song, and H. Hu, "Evaluating grain size in polycrystals with rough surfaces by corrected ultrasonic attenuation," Ultrasonics 78, 23-29 (2017).

${ }^{8}$ E. P. Papadakis, "Ultrasonic attenuation caused by scattering in polycrystalline media," in Physical Acoustics, Vols. IV-Part B, edited by W. P. Mason (Academic Press, New York and London, 1968), pp. 269-328.

${ }^{9}$ A. Bhattacharjee, A. L. Pilchak, O. I. Lobkis, J. W. Foltz, S. I. Rokhlin, and J. C. Williams, "Correlating ultrasonic attenuation and microtexture in a near-alpha titanium alloy," Metall. Mater. Trans. A 42, 2358-2372 (2011).

${ }^{10}$ A. L. Pilchak, J. Li, and S. I. Rokhlin, "Quantitative Comparison of Microtexture in Near-Alpha Titanium Measured by Ultrasonic Scattering and Electron Backscatter Diffraction," Metall. Mater. Trans. A 45, 4679-4697 (2014). 
${ }^{11}$ F. E. Stanke and G. S. Kino, "A unified theory for elastic wave propagation in polycrystalline materials," J. Acoust. Soc. Am. 75, 665-681 (1984).

${ }^{12}$ J. B. Keller, "Stochastic equations and wave propagation in random media," in Proceedings of Symposia in Applied Mathematics. Stochastic Processes in Mathematical Physics and Engineering, Vol. XVI, edited by R. Bellman (American Mathematical Society, Providence, RI, 1964), pp. 145-170.

${ }^{13}$ F. C. Karal and J. B. Keller, "Elastic, electromagnetic, and other waves in a random medium," J. Math. Phys. 5, 537-547 (1964).

${ }^{14}$ R. L. Weaver, "Diffusivity of ultrasound in polycrystals," J. Mech. Phys. Solids 38, 55-86 (1990).

${ }^{15}$ U. Frisch, "Wave propagation in random media," in Probabilistic Methods in Applied Mathematics, edited by A. Bharucha-Reid (Academic Press, New York, 1968), pp. 75-198.

${ }^{16}$ S. I. Rokhlin, J. Li, and G. Sha, "Far-field scattering model for wave propagation in random media," J. Acoust. Soc. Am. 137, 2655-2669 (2015).

${ }^{17} \mathrm{~J}$. Li and S. I. Rokhlin, "Propagation and scattering of ultrasonic waves in polycrystals with arbitrary crystallite and macroscopic texture symmetries," Wave Motion 58, 145-164 (2015).

${ }^{18} \mathrm{~J}$. A. Turner, "Elastic wave propagation and scattering in heterogeneous anisotropic media: Textured polycrystalline materials," J. Acoust. Soc. Am. 106, 541-552 (1999).

${ }^{19} \mathrm{M}$. Calvet and L. Margerin, "Velocity and attenuation of scalar and elastic waves in random media: A spectral function approach," J. Acoust. Soc. Am. 131, 1843-1862 (2012).

${ }^{20}$ L. Yang, J. Li, O. I. Lobkis, and S. I. Rokhlin, "Ultrasonic propagation and scattering in duplex microstructures with application to titanium alloys," J. Nondestruct. Eval. 31, 270-283 (2012).

${ }^{21}$ L. Yang, J. Li, and S. I. Rokhlin, "Ultrasonic scattering in polycrystals with orientation clusters of orthorhombic crystallites," Wave Motion 50, 1283-1302 (2013).

${ }^{22}$ C. M. Kube, "Iterative solution to bulk wave propagation in polycrystalline materials," J. Acoust. Soc. Am. 141, 1804-1811 (2017).

${ }^{23}$ A. Van Pamel, G. Sha, M. J. S. Lowe, and S. I. Rokhlin, "Numerical and analytic modelling of elastodynamic scattering within polycrystalline materials," J. Acoust. Soc. Am. 143, 2394-2408 (2018).

${ }^{24}$ G. Sha, M. Huang, M. J. S. Lowe, and S. I. Rokhlin, "Attenuation and velocity of elastic waves in polycrystals with generally anisotropic grains: analytic and numerical modelling," J. Acoust. Soc. Am. 147, 2422-2465 (2020).

${ }^{25}$ L. Yang and J. A. Turner, "Attenuation of ultrasonic waves in rolled metals," J. Acoust. Soc. Am. 116, 3319-3327 (2004).

${ }^{26}$ A. Van Pamel, C. R. Brett, P. Huthwaite, and M. J. S. Lowe, "Finite element modelling of elastic wave scattering within a polycrystalline material in two and three dimensions," J. Acoust. Soc. Am. 138, 2326-2336 (2015).

${ }^{27}$ A. Van Pamel, G. Sha, S. I. Rokhlin, and M. J. S. Lowe, "Finite-element modelling of elastic wave propagation and scattering within heterogeneous media," Proc. R. Soc. A Math. Phys. Eng. Sci. 473, 1-21 (2017).

${ }^{28}$ M. Ryzy, T. Grabec, P. Sedlák, and I. A. Veres, "Influence of grain morphology on ultrasonic wave attenuation in polycrystalline media," J. Acoust. Soc. Am. 143, 219-229 (2018).

${ }^{29} \mathrm{~S}$. Ahmed and R. B. Thompson, "Attenuation of ultrasonic waves in cubic metals having 
elongated, oriented grains," Nondestruct. Test. Eval. 8-9, 525-531 (1992).

${ }^{30}$ U. F. Kocks, C. N. Tomé, and H.-R. Wenk, "Texture and anisotropy: preferred orientations in polycrystals and their effect on materials properties," (Cambridge University Press, 1998).

${ }^{31}$ L. Yang, O. I. Lobkis, and S. I. Rokhlin, "Shape effect of elongated grains on ultrasonic attenuation in polycrystalline materials," Ultrasonics 51, 697-708 (2011).

${ }^{32}$ L. Yang, O. I. Lobkis, and S. I. Rokhlin, "An integrated model for ultrasonic wave propagation and scattering in a polycrystalline medium with elongated hexagonal grains," Wave Motion 49, 544-560 (2012).

${ }^{33} \mathrm{M}$. Calvet and L. Margerin, "Impact of grain shape on seismic attenuation and phase velocity in cubic polycrystalline materials," Wave Motion 65, 29-43 (2016).

${ }^{34} \mathrm{M}$. Calvet and L. Margerin, "Shape preferred orientation of iron grains compatible with Earth's uppermost inner core hemisphericity," Earth Planet. Sci. Lett. 481, 395-403 (2018).

${ }^{35}$ Y. Guo, R. B. Thompson, D. K. Rehbein, F. J. Margetan, and M. Warchol, "The effects of microstructure on the response of aluminium E-127 calibration standards," in Review of Progress in Quantitative Nondestructive Evaluation, Vol. 18, edited by D. O. Thompson and D. E. Chimenti (Kluwer Academic/Plenum Publishers, 1998), pp. 2337-2344.

${ }^{36}$ E. P. Papadakis, "The measurement of ultrasonic attenuation," in Physical Acoustics, Vol. XIX, edited by R. N. Thurston and A. D. Pierce (Academic Press, San Diego, CA, 1990), pp. 107-155. ${ }^{37}$ G. Sha and S. I. Rokhlin, "Universal Scaling of Transverse Wave Attenuation in Polycrystals," Ultrasonics 88, 84-96 (2018).

${ }^{38}$ M. Huang, G. Sha, P. Huthwaite, S. I. Rokhlin, and M. J. S. Lowe, "Elastic wave velocity dispersion in polycrystals with elongated grains: theoretical and numerical analysis," J. Acoust. Soc. Am. 148, 3645-3662 (2020).

${ }^{39}$ S. M. Rytov, Y. A. Kravtsov, and V. I. Tatarskii, "Principles of statistical radiophysics 4: wave propagation through random media," (Springer, 2011).

${ }^{40}$ R. C. Bourret, "Stochastically perturbed fields, with applications to wave propagation in random media," Nuovo Cim. Ser. 10 26, 1-31 (1962).

${ }^{41}$ S. A. Shapiro and G. Kneib, "Seismic attenuation by scattering: Theory and numerical results," Geophys. J. Int. 114, 373-391 (1993).

${ }^{42} \mathrm{M}$. Norouzian and J. A. Turner, "Ultrasonic wave propagation predictions for polycrystalline materials using three-dimensional synthetic microstructures: Attenuation," J. Acoust. Soc. Am. 145, 2181-2191 (2019).

${ }^{43}$ C.-S. Man, R. Paroni, Y. Xiang, and E. A. Kenik, "On the geometric autocorrelation function of polycrystalline materials," J. Comput. Appl. Math. 190, 200-210 (2006).

${ }^{44}$ MATLAB, "version 9.6.0 (R2019a)," (The MathWorks Inc., Natick, Massachusetts, 2019).

${ }^{45}$ J. V. Noble, "Gauss-Legendre principal value integration," Comput. Sci. Eng. 2, 92-95 (2000).

${ }^{46}$ R. Quey, P. R. Dawson, and F. Barbe, "Large-scale 3D random polycrystals for the finite element method: Generation, meshing and remeshing," Comput. Methods Appl. Mech. Eng. 200, 1729-1745 (2011).

${ }^{47}$ M. Huang, G. Sha, P. Huthwaite, S. I. Rokhlin, and M. J. S. Lowe, "Maximizing the accuracy of finite element simulation of elastic wave propagation in polycrystals," J. Acoust. Soc. Am. 148, 1890-1910 (2020). 
${ }^{48}$ S. I. Rokhlin, D. E. Chimenti, and P. B. Nagy, "Physical ultrasonics of composites," (Oxford University Press, New York, NY, 2011).

${ }^{49} \mathrm{P}$. Huthwaite, "Accelerated finite element elastodynamic simulations using the GPU," J. Comput. Phys. 257, 687-707 (2014).

${ }^{50}$ A. A. Istratov and O. F. Vyvenko, "Exponential analysis in physical phenomena," Rev. Sci. Instrum. 70, 1233-1257 (1999).

${ }^{51}$ S. I. Ranganathan and M. Ostoja-Starzewski, "Universal elastic anisotropy index," Phys. Rev. Lett. 101, 3-6 (2008).

${ }^{52}$ R. C. Bourret, "Ficton theory of dynamical systems with noisy parameters," Can. J. Phys. 43, 619-639 (1965). 